Investigation of Passive Flow Control

Techniques to Enhance the Stall

Characteristics of a Microlight Aircraft

by

Hery A Mwenegoha and Mark Jabbal

Reprinted from

International Journal of Flow Control

Volume 5 · Number 3 \& 4 · September/December 2013

Multi-Science Publishing

ISSN 1756-8250 


\title{
Investigation of Passive Flow Control Techniques to Enhance the Stall Characteristics of a Microlight Aircraft
}

\author{
Hery A Mwenegoha ${ }^{1}$ and Mark Jabbal ${ }^{2}$ \\ Brunel University, School of Engineering and Design, London, UB8 3PH, United Kingdom
}

Received date 6/9/2013; Accepted date 30/12/2013

\begin{abstract}
This report investigates the enhancement of aerodynamic stall characteristics of a Skyranger microlight aircraft by the use of passive flow control techniques, namely vortex generators and turbulators. Each flow control device is designed and scaled to application conditions. Force balance measurements and surface oil flow visualisation are carried out on a half-model of the microlight to further investigate the nature of the flow on the aircraft with and without the flow control devices. The results indicate a clear advantage to the use of turbulators compared with vortex generators. Turbulators increased the maximum lift coefficient by $2.8 \%$, delayed the onset of stall by increasing the critical angle by $17.6 \%$ and reduced the drag penalty at both lower (pre-stall) and higher angles of attack by $8 \%$ compared to vortex generators. With vortex generators applied, the results indicated a delayed stall with an increase in the critical angle by $2 \%$ and a reduced drag penalty at higher angles of attack.
\end{abstract}

Key Words: microlight; stall; flow control; vortex generator; turbulator; wind tunnel

\section{INTRODUCTION}

This project is an investigation of passive flow control techniques to enhance the stall characteristics of an in-house Skyranger microlight. Increased performance and safety gives assurance to the airworthiness of any aircraft and especially to 'self-build' aircraft like the Skyranger microlight due to the increased variability in these projects. The Skyranger (Figure 1) falls under the category of homebuilt aircraft, as described by the British Microlight Aircraft Association [1]. It has a MTOW of $450 \mathrm{~kg}$, wing span (b) of $9.5 \mathrm{~m}$, wing chord (MAC) of $1.5 \mathrm{~m}$ and uses a general aviation aerofoil with a thicknessto-chord ratio of $15 \%$.

Passive flow control techniques have been applied over the past 50 years in the aviation industry with progressive development through increased advanced research and technology. The techniques have been applied on commercial aircraft; examples include application of vortex generators on the B737 and B767 to eliminate shock-induced separation. These devices have also been applied in general aviation aircraft, including the Gulfstream V and Piper Malibu Meridian (Figure 2) to improve the performance and safety of these aircraft. On the Piper Malibu Meridian, the flap-mounted vortex generators enabled the aircraft to easily pass the FAA safety certification requirement of a slow stall speed (below $61 \mathrm{kts}$ ) [2]. Turbulators have also been applied in general aviation aircraft; for example, use of leading edge turbulators on BioniX15 to improve the stall and landing performance [3]. However, to-date there has been scarce work to compare the relative performance of such passive flow control devices.

These devices improve the performance and stall characteristics of an aircraft by changing the nature of the airflow downstream of their location. They generate streamwise vortices which redistribute momentum across the boundary layer. In most wings, the stability of the boundary layer is affected by the increase in the adverse pressure gradient beyond the suction peak. As the angle of attack of the wing is progressively increased, the adverse pressure gradient builds up towards the trailing edge of the wing due to retardation of the mainstream flow beyond the maximum thickness region. The adverse pressure gradient enforces the viscous shear forces tending to slow the fluid even more. Further downstream, the adverse pressure gradient and the viscous shear forces are strong enough to lead to a separated and 


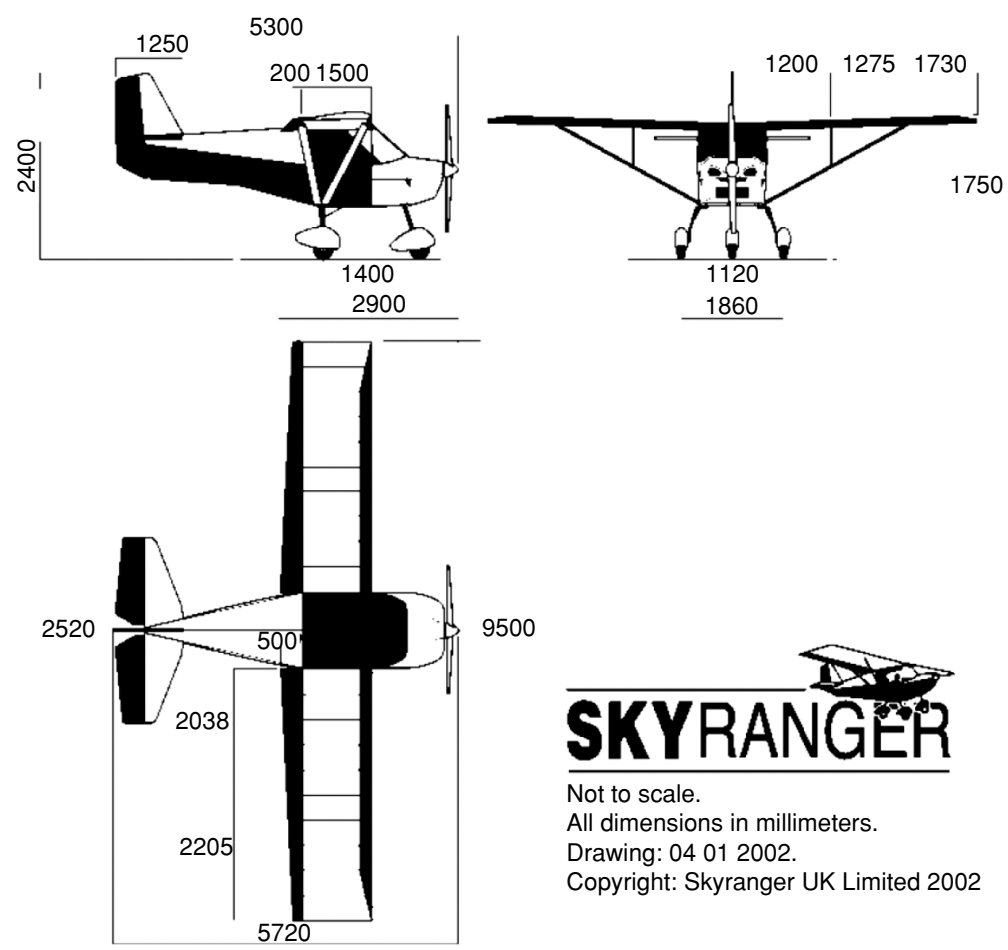

Figure 1. 3-view schematic of the Skyranger microlight.

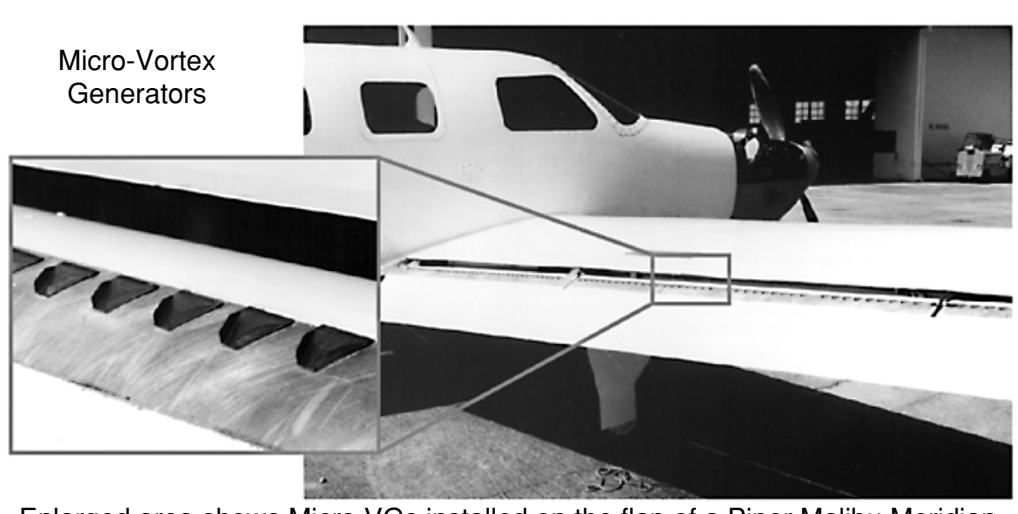

Enlarged area shows Micro VGs installed on the flap of a Piper Malibu Meridian.

Figure 2. Flap-mounted vortex generators on a Piper Malibu.

reversed flow. Therefore these devices, by the generation of vortices, shift the separation region further downstream and delay the onset of separation.

In the early stages of this work, a feasibility analysis was carried out looking at different passive flow control techniques that could be applied on the microlight. The analysis investigated vortex generators, turbulators, wing cuffs and stall fences. The feasibility study analysed the benefits in separation and stall arrest, manufacturing complexity, costs and performance parameters. It was determined that turbulators and vortex generators could offer increased benefits when applied to the microlight compared to wing cuffs and stall fences. The feasibility analysis showed that wing cuffs had more manufacturing difficulties than the other devices while stall fences were mainly used on swept wings like the T-38 Talon, hence rendering these devices unsuitable for application on the microlight Skyranger.

The work aims at improving the performance and stall characteristics of the Skyranger microlight by a wind tunnel study (force balance measurements and surface oil flow visualisation) of vortex 
generators and turbulators using a half model of the aircraft. It is expected that both the vortex generators and turbulators will increase the maximum lift coefficient, make the stall more gradual, decrease the stall rate, increase the critical angle of attack, reduce the overall drag and increase the efficiency at a drag penalty governing the performance parameter of each device. Based on a cost/benefit analysis, it is anticipated that this report will provide a definitive assessment of the most suitable type of passive flow control device to be used on a microlight.

\section{THEORETICAL ANALYSIS}

This section involves the study of the aerofoil and wing of the microlight using Javafoil and XFLR5 respectively. This study will quantify the theoretical stall characteristics without the devices and will subsequently be used in the design of the flow control devices (Section 3).

\subsection{Skyranger Geometry}

Measured values of the microlight included the wing mean chord, $\bar{c}=1.5 \mathrm{~m}$, wing span $\mathrm{b}=9.5 \mathrm{~m}$, and a fuselage length of $5.3 \mathrm{~m}$ and height $2.4 \mathrm{~m}$. Calculated wing area was $17.55 \mathrm{~m}^{2}$, stall speed $V_{s}$ was 32 knots. Aerofoil coordinates were measured and normalized. The microlight was scaled down by a factor of 10.5:1 to make the half-model microlight. The operating Reynolds numbers were 149,950 and $2.36 \infty 10^{6}$ for the model and full-scale microlight respectively based on the cruise speed and mean aerodynamic chord. The difference in the Reynolds number of the model and the full scale brings about Reynolds scaling effects, which will be discussed further herein.

\subsection{Aerodynamic Analysis of the Microlight Aerofoil (2D)}

In Javafoil, a study of the aerodynamic and stall characteristics of the microlight aerofoil section using the aerofoil coordinates was carried out so as to quantify the $2 \mathrm{D}$ properties and use the results in the design of the flow control devices. In the options tab of the applet, the density was set to $1.225 \mathrm{kgm}^{-3}$ for the sea level density and aspect ratio set to 6.3 , so as to take into account the effect of the induced angle of incidence. The surface finish was set to painted fabric and the angles of attack analysed ranged from $-4^{\circ}$ to $23^{\circ}$. As the lowest Reynolds number used was 149,950 , then the empirical transition criteria selected for the solver was Eppler for $\operatorname{Re}>1 \infty 10^{5}$. This criterion follows an integration of the simplified boundary layer equations from the stagnation point towards the trailing edge by the use of $2^{\text {nd }}$ order Runge-Kutta scheme with stabilization by automatic step reduction. The results of this analysis are presented in Figure 3. The maximum lift coefficient was 1.3, and therefore the stall speed is calculated using the relation below

$$
V_{S}=\sqrt{\frac{2 W}{\rho S C_{L_{\max }}}}
$$

(a)

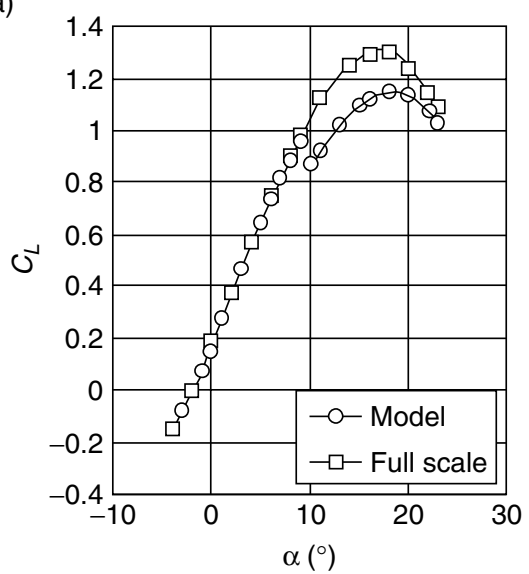

(b)

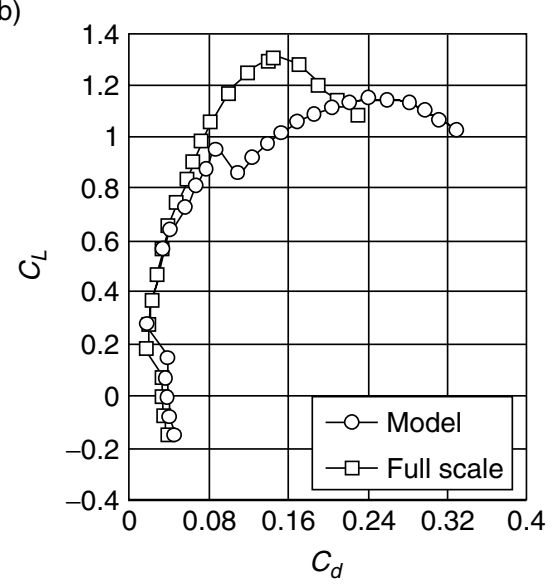

Figure 3. (a) Lift curve \& (b) Drag polar of the aerofoil section ( $\mathrm{Cl}$ vs. Cd). 
Where $V_{s}$ is $17.94 \mathrm{~ms}^{-1}$ or 33.23 knots. The observed critical angle of attack was $16^{\circ}$ for the full scale and $17^{\circ}$ for the model. The lift curve slope, $C_{L_{\alpha}}=0.087 /^{\circ}$. The observed zero lift angle of attack was $-2.21^{\circ}$ and the observed lift coefficient at zero degrees angle of attack $\left(C_{L_{0}}\right)$ was 0.1923 . From the drag polar, the drag coefficient at zero lift $C_{D_{O}}$ was 0.03 and 0.035 for the full scale and model microlight respectively.

\subsection{Aerodynamic Analysis of the Microlight Wing (3D)}

The $2 \mathrm{D}$ properties of the aerofoil section were correlated at this point with the $2 \mathrm{D}$ results from an aerofoil analysis in XFLR5. This correlation was done so as to check for any significant variations in the results and therefore help improve the design process of the flow control devices. But also using the 2D analysis in XFLR5, a 3D interpolation of the flight envelope could be achieved for the wing and plane analysis of the microlight wing. A range of Reynolds numbers was selected for this analysis, ranging from $1 \infty 10^{5}$ to $4 \infty 10^{6}$ for the $2 \mathrm{D}$ analysis so as to cover a wide flight envelope and to reduce errors in the solution. Therefore the $3 \mathrm{D}$ solution covered the flight envelope from $-4^{\circ}$ to $13^{\circ}$. This range was deemed sufficient enough to correlate with the other computational methods used. The Reynolds numbers of interest are 149,950 and $2.36 \infty 10^{6}$ for the model and full-scale aircraft respectively. With the $2 \mathrm{D}$ analysis for a Reynolds number of $2.36 \infty 10^{6}$, the maximum lift coefficient is seen to be 1.32 corresponding to a critical angle of attack of $16.5^{\circ}$ and a stall speed of $17.81 \mathrm{~ms}^{-1}$ or 33 knots.

Figure 4 of the $3 \mathrm{D}$ analysis showed $C_{L_{\alpha}}=0.07 /^{\circ}$. The observed $C_{L_{0}}$ was 0.2 and the observed incidence at zero lift, $\alpha_{0}=-2.86^{\circ}$. The discrepancy of the correlation between $2 \mathrm{D}$ and a 3D analysis is shown in the relation:

(a)

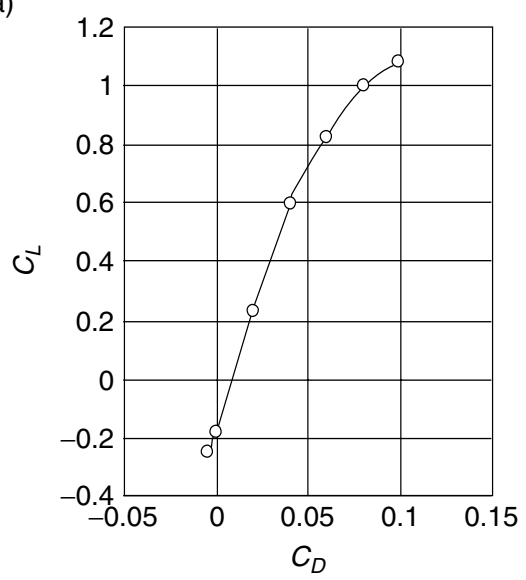

(a) Drag polar curve

(c)

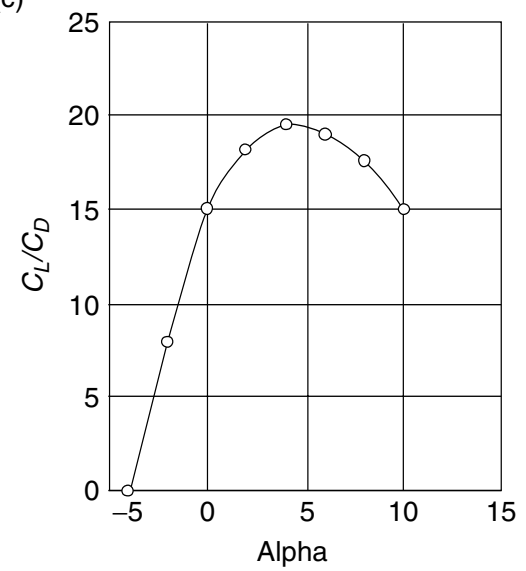

(c) Efficiency vs. angle of attack (b)

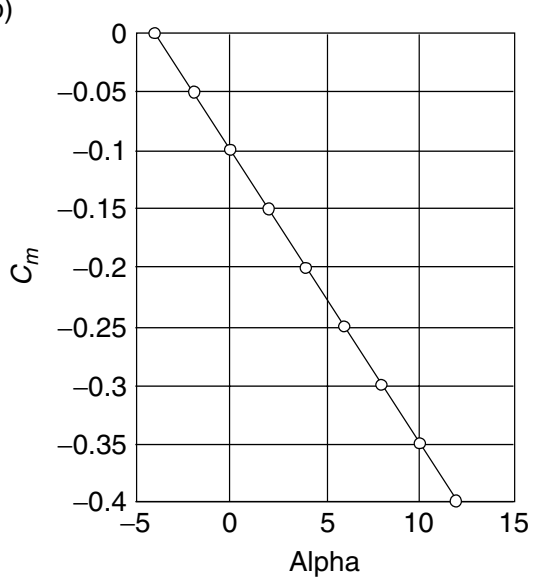

(b) Pitching moment coefficient vs. angle of attack

(d)

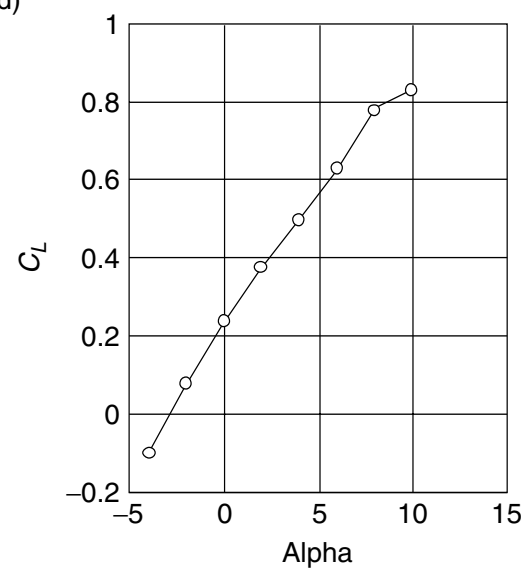

(d) Lift curve slope

Figure 4. 3D analysis results of the Skyranger microlight. 


$$
C_{L_{\alpha_{3} D}}=\frac{C_{L_{\alpha_{2 D}}}}{1+\frac{C_{L_{\alpha_{2 D}}}}{\pi(A R) e}}
$$

The observed $\mathrm{C}_{\mathrm{D} 0}$ was 0.0098 . The total drag can be estimated at a particular lift coefficient using the drag polar in Figure 4 and can be generated using the equation

$$
C_{D}=C_{D_{0}}+k C_{L}^{2}
$$

For a $C_{L}$ of 0.4 corresponding to a $C_{D}$ of 0.02 , gives $\mathrm{k}=0.0625$, where $\left.\mathrm{k}=\pi(A R)\right]^{-1}$. For the microlight wing section; AR of 6.3 gives $\mathrm{e}=0.84$. This can be used for further reference for conversion of the 2D lift curve slope to the 3D lift curve slope for the Skyranger. A tangent line passing through the origin of the axis and touching a point on the drag polar gives the point of maximum lift-to-drag ratio, $L / D$. For this case, this tangent is at a $C_{L}=0.2$ and $C_{D}=0.02$ giving $\left(C_{L} / C_{D}\right)_{\max }=20$. When compared to the $L / D$ curve on Figure 4 , it can be seen that this occurs at an angle of attack of about $4^{\circ}$.

From the stability curve in Figure 4, it can be seen that the microlight has a longitudinal stability shown by the negative slope of the pitching moment coefficient curve. It is also seen that, the microlight has a $C_{M_{\alpha=0}}=-0.1$ and $\mathrm{C}_{M_{\alpha}}=-0.025 /^{\circ}$ giving an angle of attack at zero pitching moment of $-4^{\circ}$. The estimated pitching moment $\stackrel{\alpha}{c}$ urve equation for the microlight is

$$
C_{M}=-0.025 \alpha-0.1
$$

As $\alpha_{\mathrm{o}}=-2.86^{\circ}$, the pitching moment at zero lift, $C_{M_{0}}=0.0285$. This is equivalent to the pitching moment coefficient about the aerodynamic centre of the model microlight. As the aerodynamic centre is the aft-limit of the centre of gravity for positive longitudinal position, then it can be estimated, as subsequently shown in the results section.

\subsection{Reynolds' Scaling Effect}

Trip strips are artificial roughness added to the model so as to fix the transition from a laminar to a turbulent boundary layer profile on the model. These trip-strips introduced are applied on the model microlight so as to reduce the effects associated with operating the model at a different Reynolds number from the full-scale aircraft as earlier described. Higher Reynolds number suggests that transition will occur earlier and separation delayed.

Therefore the artificially induced transition of the laminar flow over the model at the same points where transition occurs on the full-scale microlight matches the behaviour of the flow over the model to the full-scale microlight. From Figure 5 it is shown that transition on the full scale aerofoil with a Reynolds number of $2.36 \infty 10^{6}$ will occur at a location $23 \%$ of the mean aerodynamic chord $(x / c=0.23)$ for angles of attack in the rage of $-4^{\circ}$ to $2^{\circ}$ and on average at $2 \%$ of mean aerodynamic chord $(\mathrm{x} / \mathrm{c}=0.02)$ for angles of attack greater than $3^{\circ}$. Thus the trip-strip is to be located at the latter chord wise position on the model so as to reduce the effects of Reynolds scaling as shown in Figure 5.

For this model, the traditional trip strip method based on the use of grit particles on a tape (abrasive tape) is used. When properly applied, this gives a three-dimensional trip, which simulates a natural transition occurring on the model but rather at a fixed position than occurring over a stream wise length. The height of the trip-strip was estimated using the equation described by "Braslow and Knox" [4].

$$
h=\frac{12 K}{\operatorname{Re}_{f t}}
$$

Where, $h$ is the height of the grit particles in inches, $\operatorname{Re}_{f t}$ is the Reynolds number per foot based on the free stream speed, $K$ is a constant (critical roughness Reynolds number) based on grit roughness. The critical roughness Reynolds number, $\mathrm{Re}_{K}$ can be determined using the equation 
(b)

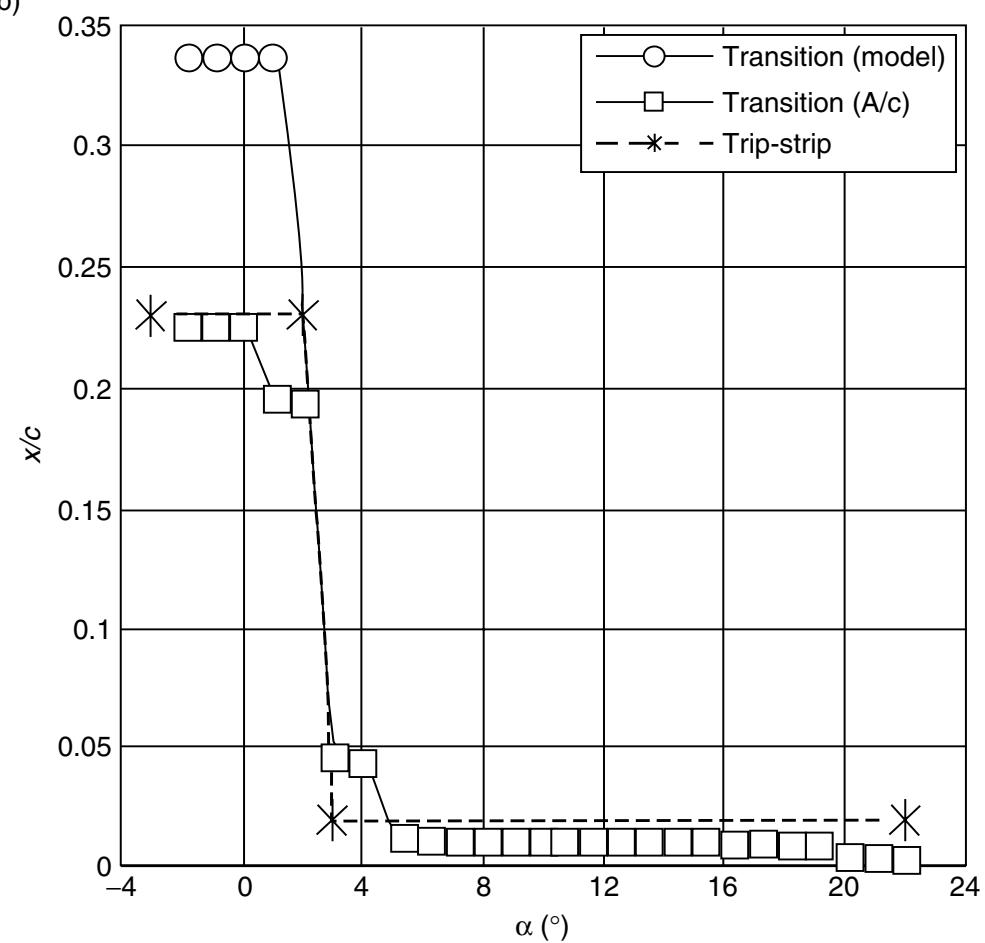

Figure 5. Transition of the boundary layer from Javafoil analysis and trip strip location.

$$
\operatorname{Re}_{K}=\frac{V_{K} K^{\prime}}{v_{K}}
$$

Where, $V_{K}$ is the velocity at height $K^{\prime}, K^{\prime}$ is the grit diameter and $v_{K}$ is the kinematic viscosity at $K^{\prime}$. Determination of $\operatorname{Re}_{k}$ is usually made experimentally and $K$ has been found to be 600 for Reynolds number greater than 100,000 (based on free stream speed and distance from the leading edge to the tripstrip) and $K$ is 1000 for Reynolds number less than 100,000 [4]. Roughness Reynolds number is presented in Table 1. Therefore for the model operating at a wind tunnel velocity of $20 \mathrm{~ms}^{-1}, \mathrm{Re}_{f t}=415,604 / \mathrm{ft}$ of the chord, which leads to a trip strip with grit particles of height 0.0288 inches or $0.73 \mathrm{~mm}$. As at both positions, the corresponding Reynolds numbers were less than 100,000 therefore $K=1000$ was used.

Grit particles with a grit number P30 with a height of 0.028 inches would have been desirable but were not readily available. A P40 $(0.02$ inches $\sim 0.5 \mathrm{~mm})$ abrasive tape was the closest grit size readily available and therefore used; the shortfall in thickness was compensated for by the use of double-sided tape with a thickness of $0.1 \mathrm{~mm}$. The only difficulty in having a layer of double sided tape is its tendency to trip the flow two dimensionally. As such, the trip-strip was applied over a width of $5 \mathrm{~mm}$, as suggested by Pope et al [4]. The width accounts for the transition region which is not a single point but rather a finite distance. This region has 3 stages: a linear stage; non-linear stage with 2D Tollmien Schlichting waves and a 3D stage marking complete transition.

\subsection{XFLR5 and JavaFoil Validation}

XFLR5 and JavaFoil used in studying the clean wing of the model and in the design process of the flow control devices have their weaknesses and strengths when compared to actual experimental results. The

Table 1. Roughness Reynolds number based on stream wise location of the trip strip and free stream velocity

\begin{tabular}{llllll}
$\alpha$ & $\mathbf{x} / \mathbf{c}$ & $\boldsymbol{V}\left(\mathbf{m s}^{-\mathbf{1}}\right)$ & $\rho\left(\mathbf{k g m}^{-\mathbf{3}}\right)$ & $\boldsymbol{v}\left(\mathbf{m}^{\mathbf{2}} \mathbf{- 1}\right)$ & $\mathbf{R e}$ \\
\hline$-4^{\circ} \leq \alpha \leq 2^{\circ}$ & 0.23 & 20 & 1.225 & $1.43 \infty 10^{5}$ & 35384 \\
\hline$>3^{\circ}$ & 0.02 & 20 & 1.225 & $1.43 \infty 10^{5}$ & 3077 \\
\hline
\end{tabular}


weaknesses mainly arise from the limitations of numerical methods to accurately predict the non-linear behaviour of most aerodynamic properties

With Javafoil, a NACA 4415 airfoil had been analysed and the results compared to wind tunnel measurements in a Laminar Wind Tunnel at Stuttgart [5]. The data is presented in NACA Report No. 824 and computational results were produced by both XFLR5, implementing XFOIL algorithms, and JavaFoil. At a Reynolds number of $3 \times 10^{6}$, both methods overpredicted the maximum lift coefficient. XFLR5 overpredicted $C_{L \max }$ by $28.5 \%$ while JavaFoil overpredicted $C_{L \max }$ by $33 \%$. The lift-curve slope was also overpredicted in JavaFoil because the boundary layer displacement effects were not modeled while XFLR5 models the effects. Both numerical methods were seen to properly predict the zero-lift angle. For lower angles, JavaFoil closely approximated the drag values, but the values were considerably higher than the XFLR5 prediction. For example JavaFoil, predicted a $C_{D O}$ that was $25 \%$ greater than experiment while XFLR5 predicted a $C_{D O}$ that was $6.25 \%$ lower than the experimental value [5].

XFLR5 is used for both 2D and 3D analysis of the model in the present study. The 2D results were used for the interpolation of the 3D results. The Vortex Lattice Method (VLM) was used in the numerical approximation of the aerodynamic coefficients for the 3D analysis. Other numerical schemes in the package include Lifting Line theory (LLT) and 3D panel method. A validation experiment was conducted using a test sail plane, and the measured results were compared to the XFLR5 predicted results with and without the body [6]. The results showed that all the numerical schemes correctly predicted the value of the zero-lift angle and that the LLT method best fits the non-linearity of the lift curve slope. Also the numerical schemes underestimated the total drag and gave good predictions of $C_{M O}$ with an adequate moment slope. Modelling the body was seen to generate numerical noise due to the difficulty to model connections between the wing and the body.

With the presented results, XFLR5 is observed to accurately predict $C_{L O}$ and the zero lift angle. The lift-curve slope is underpredicted by $25 \%$ while the pitching moment curve is observed to be adequately predicted.

\section{FLOW CONTROL DEVICE DESIGN AND MANUFACTURE}

\subsection{Vortex Generators}

Vortex generators act like small, low aspect ratio wings placed at a configured incident angle to the incoming flow $(\beta)$, in which flow past the tip creates longitudinal streamwise vortices (Figure 6). These
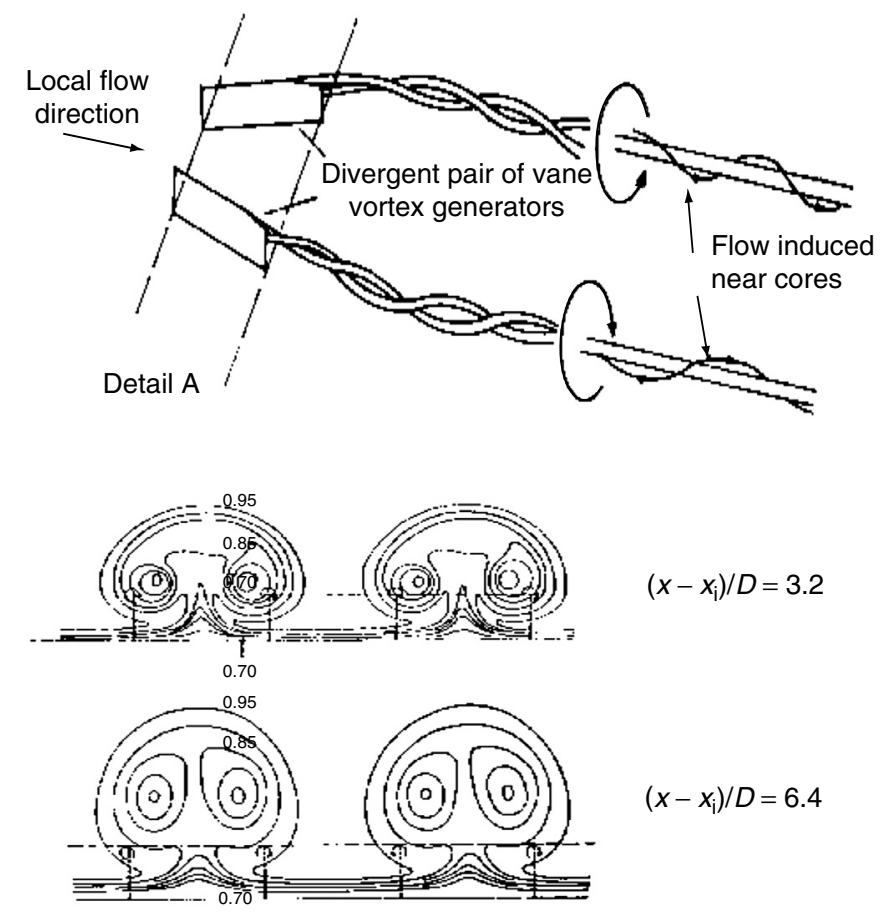

Figure 6. Vortex generator flow physics. 
vortices mix the high momentum fluid from the upper layers of the boundary layer down into the lower layers [7], and this momentum redistribution tends to shift the separation point further downstream. They also, in conjunction with other high lifting devices like flaps and slats, increase the lifting ability of the wings mainly during landing and take-off where the aircraft is operating at low speeds and high angles of attack so stalling from flow separation is of primary concern. They improve high Mach pitch characteristics beyond initial buffet and on the Boeing 767, they were used to increase stick forces per g-loading and hence provided increased buffet intensity as pilot pulled into these conditions [8]. Vortex generators tend to decrease the separation velocity at the separation region (inflection point) and therefore increase the surface static pressure. The static pressure governs all other pressures in the separation region. The increase in the surface static pressure tends to decrease the drag by increasing the back pressure which generally has two advantages; first is that the narrowed separation region tends to reduce the drag by narrowing the wake and secondly the increase in static pressure tends to reduce the pressure drag [9].

The effectiveness of vortex generators is governed by a number of parameters that are dependent on the height of the device. This height is governed by the boundary layer thickness on a particular surface optimized for effective flow control. These parameters include: $h / \delta$ (height-to-boundary layer thickness ratio); $L / h$ (non-dimensional device length); $\beta$ (device airflow incident angle); $\Delta z / h$ (non-dimensional device spacing) and $\Delta x / h$ (non-dimensional streamwise distance between the vortex generators' trailing edge and the baseline separation). Conventional vortex generators with $h / \delta \sim 1$ are found to be 'over effective' as they tend to cause a 3D attached flow with recirculation pockets on the downstream position, which tends to increase drag. Therefore the most widely used vortex generators are those with heights less than the boundary layer thickness, usually in the range of $0.1 \leq h / \delta \leq 0.5$ termed as sub boundary layer vortex generators. These are seen to be effective by producing vortices to delay separation and reattach flow with less drag penalty compared to conventional vortex generators. The most effective vortex generator group are those that produce streamwise vortices of which there are several designs, as described by Lin [10]. During cruise conditions, the aircraft operates at low angles of attack and thus vortex generators lead to an increase in drag as they act as surface protuberances. Therefore optimum placement of the devices is required where the separation is fairly fixed and doesn't require covering a large downstream distance by the devices.

\subsubsection{Selection and Sizing}

The vortex generators considered for this study are counter rotating vortex generators as these mainly generate vortices that do not degrade in strength as the counter rotating pair already has a net zero vorticity and therefore satisfy the continuity equation. These vortex generators also have minimum vortex core interference and minimum dissipation when correctly placed [11]. These vortex generators are shown in Figure 6.

After selection of the vane platform arrangement, the shape of the vortex generator had to be determined. Delta-shaped vortex generators were selected to be applied and used as they could be easily manufactured with minimal design difficulties and could be easily attached on the surface of the wing. These vortex generators also have less edge interference with streamwise vortices and therefore vortex core breakdown is more infrequent; they also have a small frontal area and thus less device drag. Prior to selection of the vane platform arrangement and the shape, the vortex height, thickness, length and spacing had to be determined with the core parameter being the vortex height, which directly determines the strength of the vortices and the device drag.

In determining the vortex generator height, the boundary layer thickness had to be studied for higher angles of attack so that the vortex generator could be designed as a function of the boundary layer thickness [10], to be in the range of $0.1 \delta \leq h \leq 0.5 \delta$ for effectiveness. Javafoil was used to carry out the study on the boundary layer profile over a $2 \mathrm{D}$ section of the wing at a Reynolds number of $2.36 \times 10^{6}$, corresponding to the Reynolds number of the full-scale aircraft at normal operating cruise conditions. It was found that the boundary layer profile for higher angles of attack was not steady and led to very high values of boundary layer thicknesses of about $38 \mathrm{~mm}$ at separation. This was deemed not feasible to be used as the basis for scaling $h$ as it would lead to significant drag at lower angles of attack.

Therefore focus was on a more stable profile at $0^{\circ}$ angle of attack since it could be well approximated for a zero pressure gradient flow and therefore boundary layer equations could be used to determine the thickness. As transition occurred very close to the leading edge for positive angles of attack $(x / c=0.02)$, the flow was turbulent for the remaining chordwise distance extent. The overall flow 
therefore had a mixed boundary layer profile, of which the thickness could be approximated using

$$
\delta_{T_{t}}=\frac{0.383 x_{T_{t}}}{\left(\operatorname{Re}_{x}\right)_{T_{t}}^{0.2}} \text { and } \bar{u}=y^{-\frac{1}{n}}
$$

Of which the profile is valid for a $7^{\text {th }}$ order turbulent velocity profile plot. This thickness assumes that the turbulent boundary layer thickness grows as if it had started at some distance, $x_{T_{t}}$ ahead of transition. This is to take into account the effect of having different boundary layer growth rates for a turbulent and laminar boundary layer flow. But since using Javafoil the displacement thickness could be determined directly, the displacement thickness is converted to the boundary layer thickness using

$$
\delta_{1}=\frac{0.0479 x}{\left(\operatorname{Re}_{x}\right)^{0.2}}=0.125 \delta
$$

As seen from the boundary layer plot (Figure 7) the average boundary layer displacement thickness at this angle of attack $\left(0^{\circ}\right)$ is $1.5 \mathrm{~mm}$. From Eq. (8), this equates to an average boundary thickness of $12 \mathrm{~mm}$. Therefore a vortex generator height equivalent to $h=0.33 \delta$ is arbitrarily chosen such that for angles of attack in the range of 10 to $17^{\circ}$ the height has a minimum limit of $h=0.11 \delta$. This ensured that under all operating conditions, the devices are within the acceptable height range for micro vortex generator effectiveness, as suggested by Lin [10].

The strength of the vortices generated, $K$, is also an important factor in determining the effectiveness of the vortex generators. The strength of these vortices is not only governed by the device height but also by the orientation with respect to the incoming local flow and the position along the chord of the wing. For high vortex generator effectiveness, a value of $K \sim 1$ is desirable [8] of which $K$ is equivalent to

$$
K=\frac{K_{v}}{h U_{e}}
$$

Where $K_{v}=4 C_{L_{V}} / \pi(A R)$. From Eq. (9), the strength of the vortices is a function of the height of the vortex generator $(h)$, the free stream velocity $\left(U_{e}\right)$, and a vortex parameter that depends on the

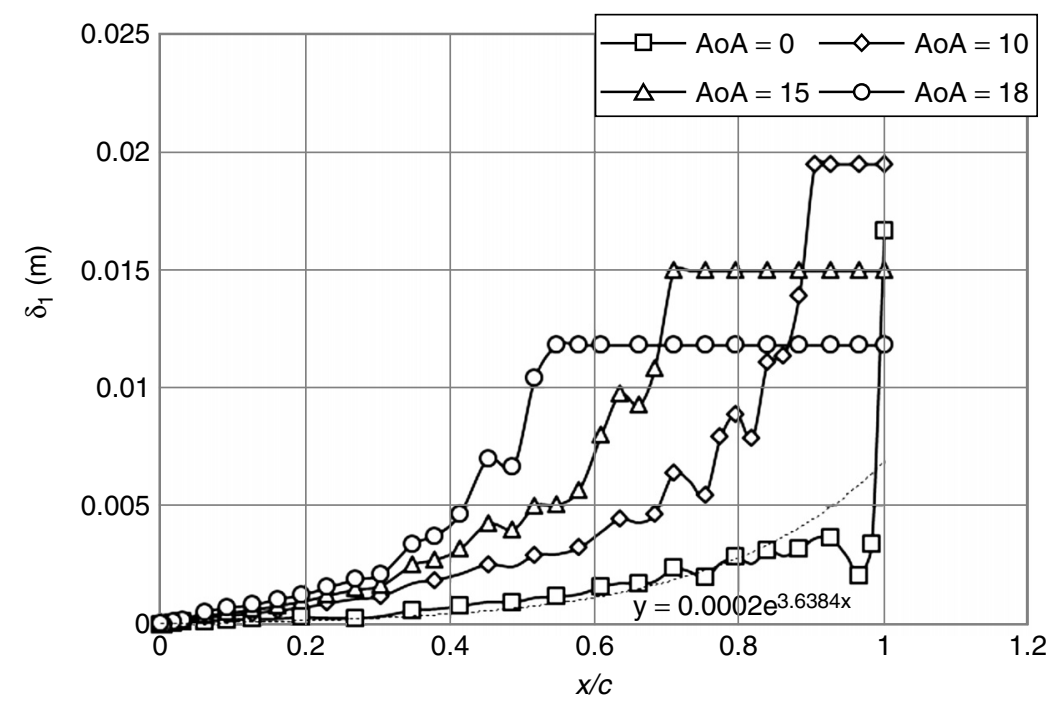

Figure 7. Boundary layer displacement thickness for different angles of attack ranging from 0 to 17 degrees. 
lift coefficient $\left(C_{L_{v}}\right)$ and the aspect ratio $\left(A R_{v}\right)$ of the vortex generator. The aspect ratio is determined by

$$
A R_{v}=\frac{4 h}{S_{v}}
$$

Since the height was determined, the aspect ratio was chosen to be 2 since the boundary layer thickness was taken at a zero pressure gradient flow and increase vortex core spacing. This led to a vortex generator length of $10 \mathrm{~mm}$. This aspect ratio was also deemed structurally better for attachment with the vortex slot because choosing anything less would have led to poor slot support. The thickness of the vortex generator was set to $t_{v} / l_{v} \leq 0.05$ as suggested by ESDU [8] applicable to delta-shaped vortex generators for effective flow control, though the thickness $\&$ edge detail have no significant effect on the flow.

The vortex generator strength parameter, $K$, had to be determined so as to determine the incidence angle, $\alpha_{v}$, of the vortex generator to the local free stream flow. This incident angle was determined at the point when the vortex generator strength parameter was approximately 1 , which is the value desired for effectiveness. The vortex generator when mirrored along the y-z plane would represent a delta wing, at a given angle of attack to the free stream. Therefore using the assumptions to derive the lift coefficient in a delta wing, the vortex generator lift coefficient was determined in accordance with ESDU [11], in which the lift is the sum of the potential flow lift and vortex lift

$$
C_{L_{v}}=K_{p} \sin \alpha_{v} \cos ^{2} \alpha_{v}+K_{V}^{\prime} \operatorname{COS} \alpha_{v} \sin ^{2} \alpha_{v}
$$

The potential flow dominates at small angles of incidence and the vortex lift dominates at higher angles of incidence. The angle of incidence for the vortex generators is the sum of the datum angle between the aircraft plane of symmetry to the free stream flow $\left(\sigma_{\text {datum }}\right)$ and the angle between the free stream flow to the local flow $(\sigma)$ and the vane setting angle $\left(\alpha_{\mathrm{v}}\right)$ given by

$$
\xi=a_{v}+\sigma_{\text {datum }}+\sigma
$$

Since there is less cross flow in the case of the unswept wing of the microlight, then the angle between the free stream flow and the local flow can be taken to be zero. $K_{p}$ and $K_{v}$ ' are functions of the aspect ratio of a delta wing for the potential and vortex flows respectively

$$
\begin{gathered}
K_{P}=1.5 A R-0.2(A R)^{2} \\
K_{v}^{\prime}=3.14+0.03 A R
\end{gathered}
$$

Therefore for an aspect ratio of 2, the values of $K_{p}$ and $K_{v}$, are determined to be 2.2 and 3.2 respectively. Further to this, for the vortex strength to be approximately 1 , the incident angle was determined to be $4-5^{\circ}$, which is less than the usual optimum of $10-15^{\circ}$ due to the high aspect ratio and the presence of sweep in the trailing edge detail. The design of the vortex generators is presented in Figure 8.

The spacing between members of the counter rotating pair $\left(d_{v}\right)$ and between different pairs $\left(D_{v}\right)$ is a function of the vortex generator height such that $d v / h=2$ and $D_{v} / h=10$, as suggested by ESDU. Therefore with a vortex height of $4 \mathrm{~mm}, D_{v}=40 \mathrm{~mm}$ and $d_{v}=8 \mathrm{~mm}$. These values will include an error of $+/-0.4 \mathrm{~mm}$ to account for the effect of the support thickness on the vortex generator height as well as the double-sided tape to be used with a thickness of $0.1 \mathrm{~mm}$. With $D_{v}$ being $40 \mathrm{~mm}$ and the wing span being $450 \mathrm{~mm}, 10$ to 11 pairs of counter rotating vortex generators would fit along the whole span.

\subsubsection{Design and Manufacture}

Solid works $2011 \times 64$ edition was used in drawing and producing the 3D CAD images of the vortex generators. The support and the vortex generator were two different pieces which could be merged 


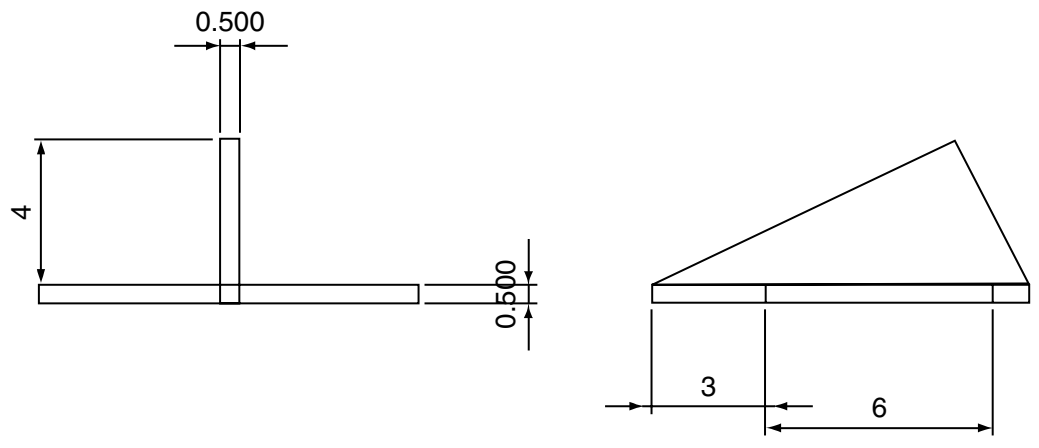

Figure 8. Vortex generator design (all dimensions in $\mathrm{mm}$ ).

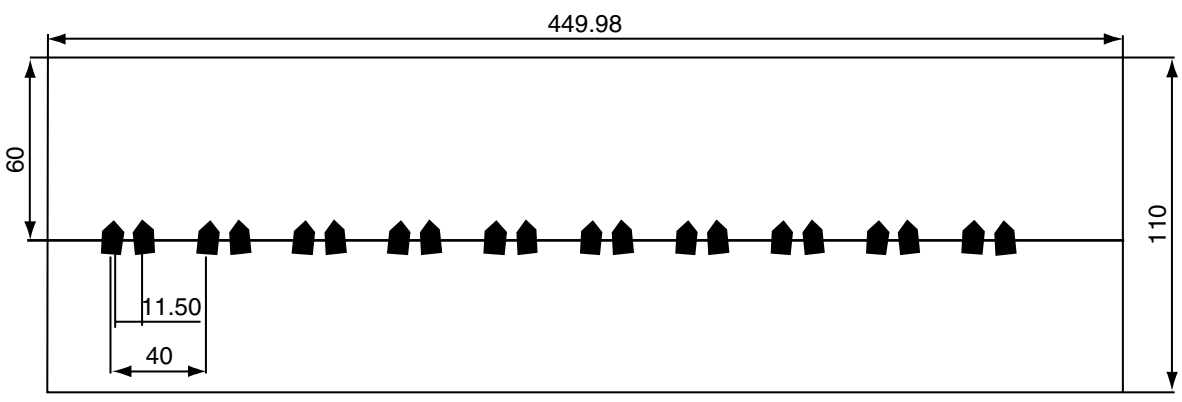

Figure 9. Arrangement of the vortex generators on the model microlight wing (all dimensions in $\mathrm{mm}$ ).

together. As laser cutting was considered the easiest and most cost effective manufacturing technique for the devices, it created a limitation in the materials to be used and therefore plywood sheets with a thickness of $0.5 \mathrm{~mm}$ were selected in manufacturing the vortex generators. The arrangement of the vortex generator array in Figure 9 was seen to be most feasible and therefore applied in the actual arrangement of the vortex generators on the wing of the model microlight Skyranger.

Using the exponential equation developed earlier for $0^{\circ}$ angle of attack (Figure 7)

$$
0.125 \delta=0.0002 e^{3.6384(x / c)}
$$

The vortex generators are applied at the region on the wing where the average boundary layer thickness of $12 \mathrm{~mm}$ lies. This region was approximately $60 \mathrm{~mm}$ behind the leading edge of the wing. This position, $60 \mathrm{~mm}$ or $\mathrm{x} / \mathrm{c}=0.55$ corresponds to a position $5 \%$ ahead of the separation line at $17.1^{\circ}$ angle of attack and therefore was theoretically deemed effective for the vortex generators.

\subsection{Turbulators}

Turbulators are passive flow control devices which tend to delay the onset of flow separation by tripping the flow into turbulent and for an existing turbulent flow, induce vortices which re-energize the flow and therefore shift the separation region further downstream. Turbulators have been significantly applied in heat conduction and radiation problems $[12,13]$ with focus being on the helical coiled turbulators and wire coiled core matrix turbulators (WCCMT) with different pitch to diameter ratios.

Attention is mainly focused on the zigzag shaped turbulators, as shown in Figure 10. Turbulators can also take the form of a single tape-strip (also called 2D turbulator), or the shape of single bumps equally spaced. For an overall benefit of the turbulator action, however, drag reduction from delaying the onset of separation should be greater than the drag due to earlier transition and the drag due to the turbulator itself [14]. Therefore turbulators are usually designed for a "low Reynolds number and high $C_{L}$ configuration" [14] as at this configuration separation occurs further upstream and therefore the turbulators will induce vortices into the flow and delay the onset of separation, therefore maximising the benefits. Turbulators are designed such that they are below the critical roughness height sufficient 


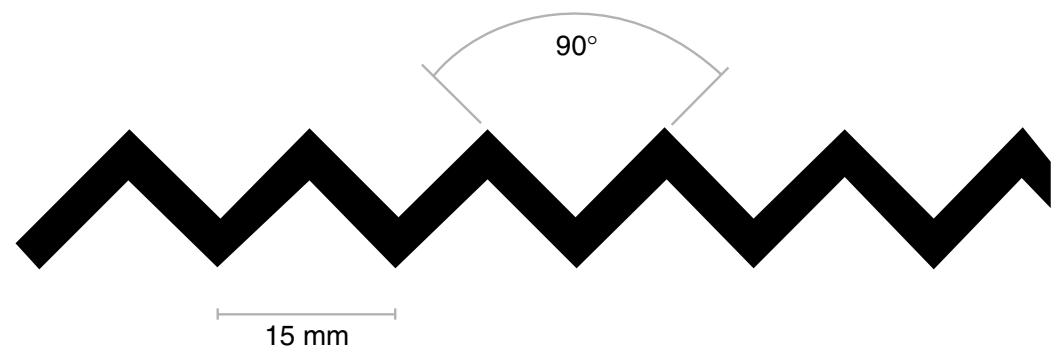

Figure 10. Zigzag shaped turbulator.

to cause boundary layer transition but not greater when operating at low Reynolds number and high $C_{L}$ configuration and therefore reducing turbulator drag. Designing turbulators for conditions of high $C_{L}$ and low Re, means when operating at low $C_{L}$ and high Re (e.g. cruise conditions) there will be a high drag penalty. Therefore a number of parameters govern their effectiveness: $h / c$ (the height-to-chord ratio determined from the local Reynolds number); $w / c$ (width-to-chord ratio for vortex growth); $\lambda$ (turbulator sweep angle or vortex spacing) and $x / c$ (distance from the trailing edge).

Most turbulators are used on winglets and wings that employ the use of aerofoils placed at different angles of attack (geometric twist or most commonly a washout) or placed according to operating configuration of a given section in terms of Re and $C_{L}$ and therefore usually applied to the inboard parts on the wing. This adds to the advantage that the outboard parts of the wings do not have to suffer from the drag penalty generated under high Re and low $C_{L}$ operating conditions as based on their design and therefore optimizing the overall efficiency of the wings. Since at high angles of attack the separation point is closer to the leading edge, then the turbulators are placed closer to the leading edge to eliminate the effect which in turn leads to more drag during a high Re and low $C_{L}$ operation.

\subsubsection{Selection and Sizing}

The turbulator height is a function of its position along the chord from the leading edge of the wing as well as the operating Reynolds number as earlier described. Since the operating Reynolds number of the full-scale aircraft was known, $2.36 \times 10^{6}$, then determination of the position of the turbulator strip was the only limiting factor. Most turbulators are made so that they can be used in controlling or inducing transition to a turbulent flow and therefore delay the onset of separation due to the high momentum turbulent flow formed after an encounter with the turbulator strip. However, for this case the idea was to control separation from an incoming turbulent flow. And therefore the position will not be upstream of the transition region but rather upstream of the separation region. Since increasing angle of attack the separation point moves further upstream, the focus is to reduce separation especially for higher angles of attack. Using Javafoil a separation plot was generated as seen in Figure 11.

For lower angles of attack, separation is seen to be at the trailing edge for the full scale aircraft but with increasing angle of attack the separation point moves further forward. It was estimated that for angles of attack in the range from 10 to $17^{\circ}$ (stall) the separation point was such that

$$
x / c=-0.0416 \alpha+1.2985
$$

From the initial analysis done it was observed that, the aircraft stalled completely at about $17^{\circ}$ to $18^{\circ}$ corresponding to a position $40 \%$ along the mean chord line from the leading edge. For increased effectiveness of the turbulators it was suggested that they should be placed 5-10\% ahead of the separation point. Therefore the turbulators were placed $10 \%$ forward of the separation point so as to optimise their efficiency at all angles of attack and especially during stalling. This position is approximately $\mathrm{x} / \mathrm{c}=0.36$ or $40 \mathrm{~mm}$ from the leading edge of the wing. Since the height of the turbulators to be employed will be significantly less than the vortex generators then the expected drag penalty resulting from the device is expected to be lower.

At this position, the boundary layer had a thickness of $6 \mathrm{~mm}$ for a $0^{\circ}$ angle of attack flow on the wing. Therefore using the tool developed by Dr. Martin Hepperle [14], the position of the turbulator was marked to be $\mathrm{x} / \mathrm{c}=0.36$. And as the local Reynolds number at the position is $6.2 \times 10^{4}$, then the height of the turbulator could be determined using the chart in Figure 12. Using the chart, the optimum 


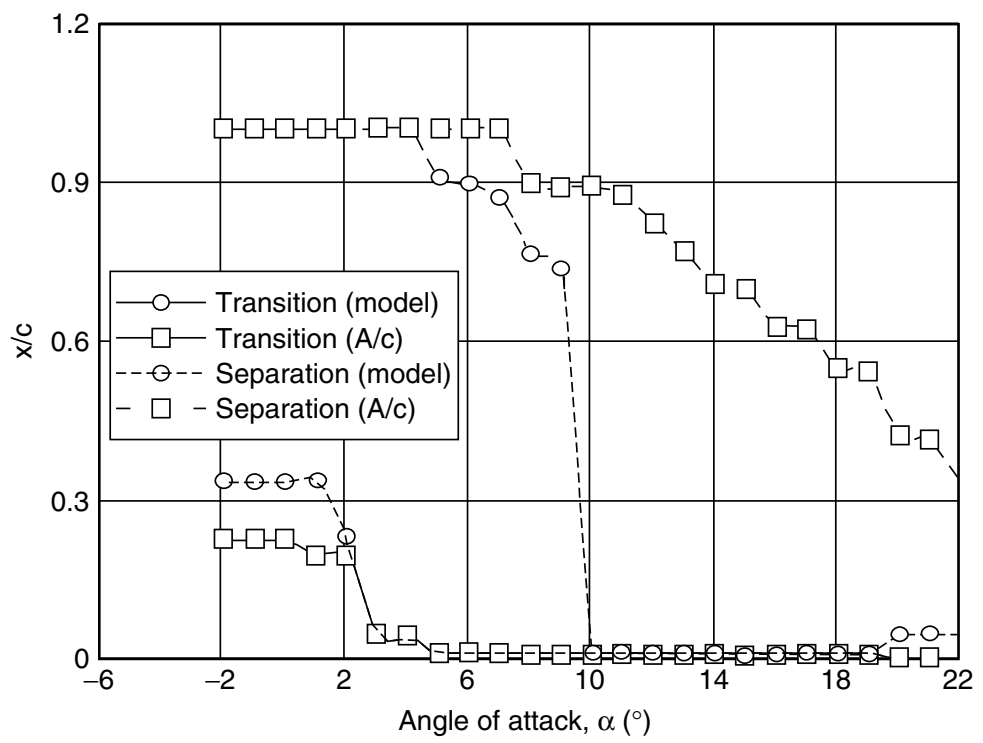

Figure 11. Transition and separation points on the full scale and model microlight.

height was found to be $h / c=0.0013$, equivalent to $0.143 \mathrm{~mm}$ for the turbulator thickness or height.

This chart can be related to the drag polar chart plotted with the separation line on the same scale and for a given lift configuration, the position of the turbulators can be estimated. This is then correlated with a polar diagram with a variation of the drag along the wing surface for a given Reynolds number and lift configuration as earlier described. This involves measuring the velocity profile at different locations along the mean chord and estimating the skin friction drag from the wall shear stress and for the given time-scale could not be done but is suggested for an optimum design of the turbulators.

Since finding information on sizing turbulators proved to be difficult, the shape and design was adopted from an earlier design by AFE [15] but with reference to the estimated height and proposed chord wise location and with a turbulator angle of $60^{\circ}$ (typically optimum). The length of the turbulator was made such that it fits the whole wingspan, $450 \mathrm{~mm}$.

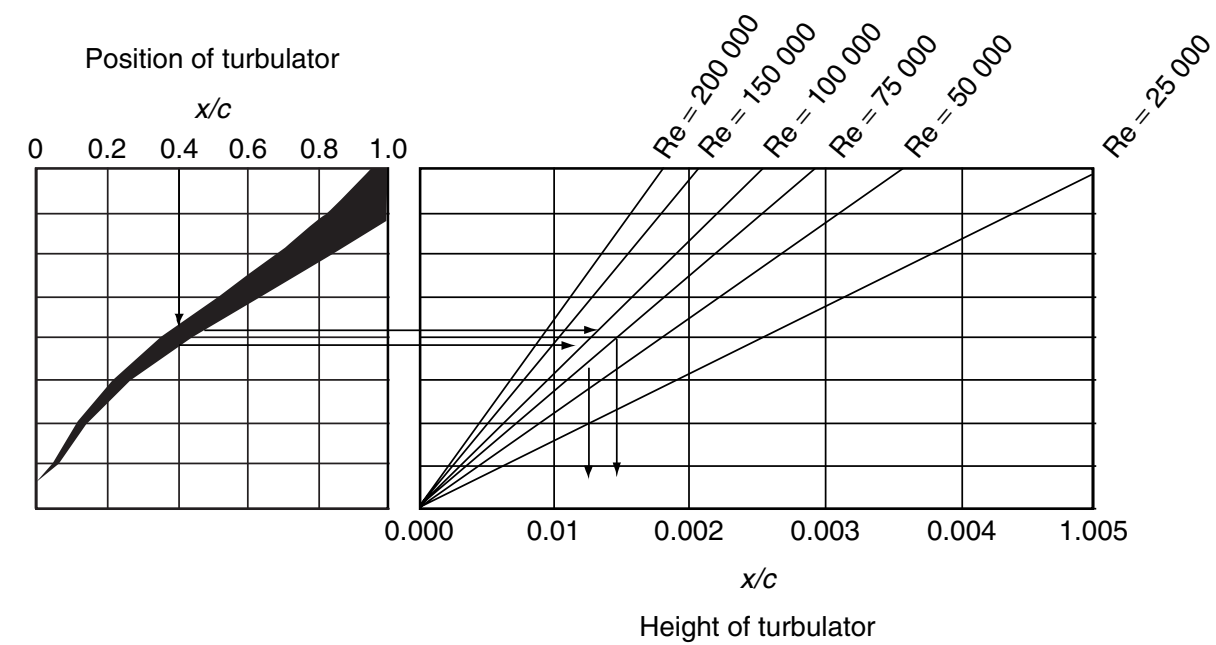

Figure 12. Turbulator sizing based on Reynolds number and turbulator chord wise position $(\mathrm{x} / \mathrm{c})[14]$. 


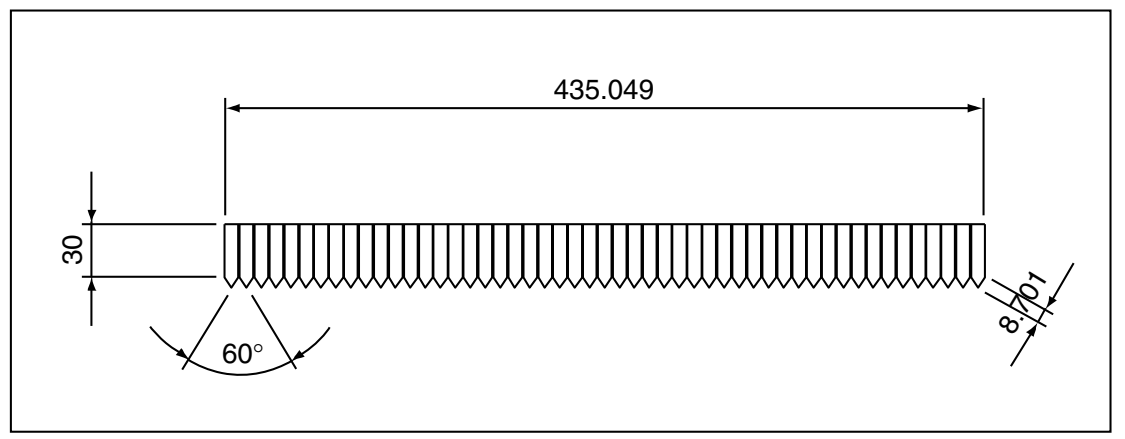

Figure 13. Turbulator strip after sketching and extrusion in Solidworks (all dimensions in $\mathrm{mm}$ ).

\subsubsection{Design and Manufacture}

Laser cutting was deemed the best manufacturing method for the device. Thin sheets of plastic and aluminium of $0.1 \mathrm{~mm}$ thickness were used to manufacture the devices. The thickness of the required turbulator strip was $0.143 \mathrm{~mm}$ therefore double-sided tape that will be used in attaching the device to the wing surface will also be used to provide the extra thickness of the device. The length of the turbulator trip was $435 \mathrm{~mm}$ of which this would leave a clearance of $15 \mathrm{~mm}$ from the fuselage so as to fully cover the wing therefore extra length came from the second turbulator. The turbulator strip used as a benchmark in this design had a length of $170 \mathrm{~mm}$ and a thickness of $0.2 \mathrm{~mm}$ with a trip angle of $60^{\circ}$ which was also adopted for this design since it offers sufficient vortex spacing. The width of the turbulator strip adopted was $30 \mathrm{~mm}$ which was also estimated using the initial turbulator strip by AFEturbulators [15] as a reference strip and was deemed viable to be applied on the wing of the half model microlight with a chord length of $110 \mathrm{~mm}$ giving a width-to-chord ratio of approximately 0.27 . The final arrangement of the turbulators is shown in Figure 13.

\section{EXPERIMENTAL APPROACH}

\subsection{Wind Tunnel}

\subsubsection{Wind Tunnel Description}

A low speed wind tunnel was used to carry out the experimental investigation of the stall characteristics of the model-microlight. The wind tunnel is a 'suction type' with a motor operating a fan providing a specific dynamic pressure at a given setting in the diffuser section. The dimensions of the test section are $0.5 \mathrm{~m} \times$ $0.5 \mathrm{~m}$. The maximum velocity of the airflow in the tunnel is $35 \mathrm{~ms}^{-1}$. The tunnel includes a three-degree of freedom force balance. This force balance had load cells which registered the different aerodynamic forces, lift, drag as well as pitching moment as a secondary property exerted on the physical model to be tested.

\subsubsection{Wind Tunnel Procedure}

The main part of this project involved the use of the force balance to measure the aerodynamic forces on the model at different configurations. After understanding the variables to be monitored (fore, aft and drag forces) during the testing phase, the force balance was calibrated so as to reduce inherent errors during measurement. Following calibration of the force balance, the test section was characterised so as to take into account the variation of the velocity from the mid-section of the test section. The observed turbulent intensity for the tunnel test-section was $0.2 \%$ [16].

To reduce errors in the measurement it is suggested by Pope, that the model's span is at most $80 \%$ of the tunnel width for 3D flow [4]. The model span was $90 \%$ of the tunnel width since it was designed for use in another prior wind tunnel. This discrepancy might lead to errors resulting from an intermediate 2D and 3D flow. The effects might lead to incorrect lift induced drag because of less cross flow or trailing vortices being generated as the model span approaches $100 \%$ of the tunnel width. Due to limited time for manufacture, the model was re-used with acknowledgment of the aforementioned errors and since the focus was mainly based on the study of flow control devices rather than to simulate free flight per se. 


\subsection{Errors in Wind Tunnel Testing}

\subsubsection{Solid Blockage}

The effect of the floor and the ceiling of the tunnel is to restrain the naturally free air curvature of the flow and therefore the model acts as one with extra camber, increasing the velocity of air. This increase in velocity (solid blockage effect) is approximated as constant over the model for customary model sizes as suggested by Pope et al [4]. Solid blockage is a function of model thickness, thickness distribution and is independent of camber [4]. The blockage only affects the stream lines further away from the model since they are more displaced and therefore the increment is less than that due to an actual area reduction. The increment in the velocity is calculated in percentages using the following equation for a 3D body [4]

$$
\varepsilon_{s h}=\frac{\Delta V}{V_{u}}=K \frac{(\text { model volume })}{C^{1.5}}
$$

Where $K$ is 0.90 for a three-dimensional wing and 0.96 for a body of revolution and $C$ is the tunnel test-section area.

\subsubsection{Wake Blockage}

The presence of the solid body in the test section of the wind tunnel will not only lead to a solid blockage effect but also a wake blockage effect in the absence of boundary layer suction control. The wake generated will have a mean velocity lower than the free stream velocity. This results in an increased velocity in the flow outside the wake boundary so as to have the same volumetric flow rate at each cross-section of the tunnel test-section. This increased velocity lowers the pressure arising from the boundary layer that grows into a wake and grows towards the model and therefore creating a pressure gradient at the model which in turn increases the velocity.

The wake blockage effect is estimated for a 3D body spanning the tunnel by

$$
\varepsilon_{w b}=\frac{\Delta V}{V_{u}}=\frac{S}{4 C} C_{D_{u}}
$$

The effects due to the solid blockage and the wake blockage can be summed up by an approximation

$$
\begin{gathered}
\varepsilon_{t}=\varepsilon_{s b}+\varepsilon_{w b} \\
\varepsilon_{t}=\frac{1}{4} \frac{\text { model frontal area }}{\text { test section area }}
\end{gathered}
$$

Using the equation above, the total blockage was estimated for each angle of attack using the velocity registered at each configuration. This effect is plotted and presented in Figure 14. It was generally observed that the blockage effect increased significantly at higher angles of attack. This was due to the increased growth rate of the wake at higher angles of attack. The total blockage effect is less than $5 \%$ at higher angles of attack. The total blockage can be estimated by the relation given below

$$
\varepsilon_{t}=\left(8 \times 10^{-20}\right) \alpha^{6}+\left(9 \times 10^{-9}\right) \alpha^{5}+\left(9 \times 10^{-8}\right) \alpha^{4}+\left(4 \times 10^{-6}\right) \alpha^{3}+\left(8 \times 10^{-5}\right) \alpha^{2}+0.006 a+0.014
$$

\subsubsection{Force Balance Measurement Uncertainty}

The observed errors during the measurement of the aerodynamic forces on the model microlight are presented in Table 2. The values used in the analysis involve three test runs using the model microlight with the average of these values used, taking into account the atmospheric conditions at a given time. For a given angle of attack

$$
V_{C A S}=V_{\bullet}\left(1+\varepsilon_{t}\right)
$$




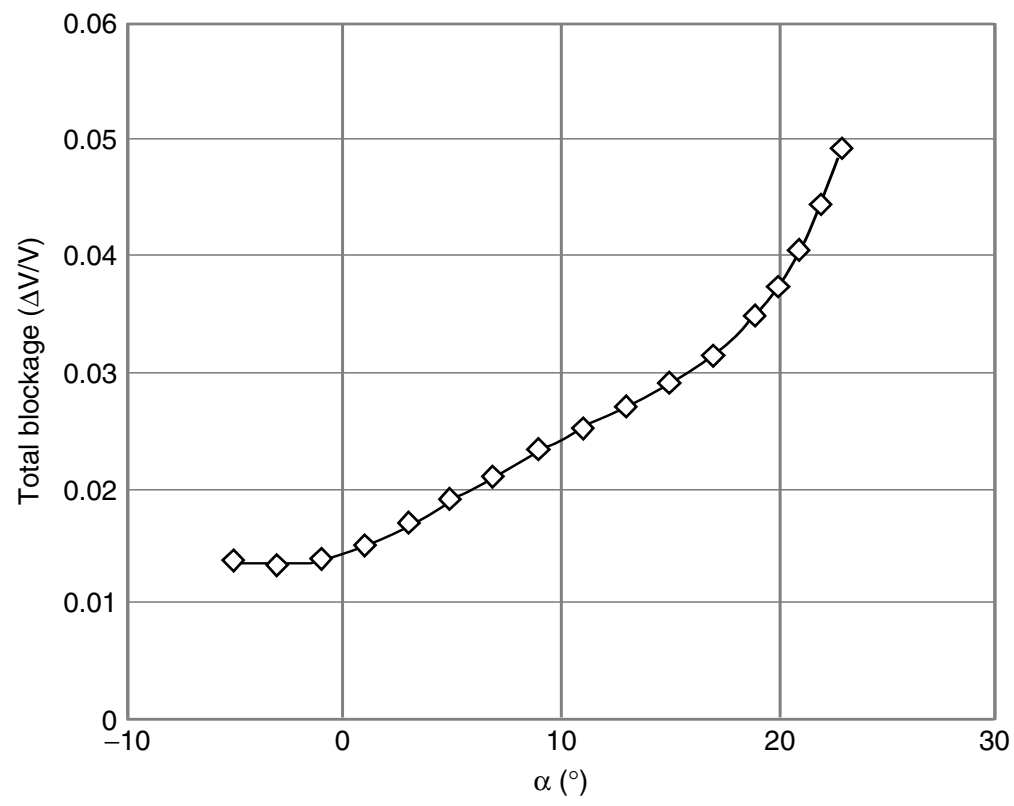

Figure 14. Total blockage effect caused by the models panning the test section at different angles of attack.

Table 2. Observed errors of force balance measurements at $20 \mathrm{~ms}^{-1}$

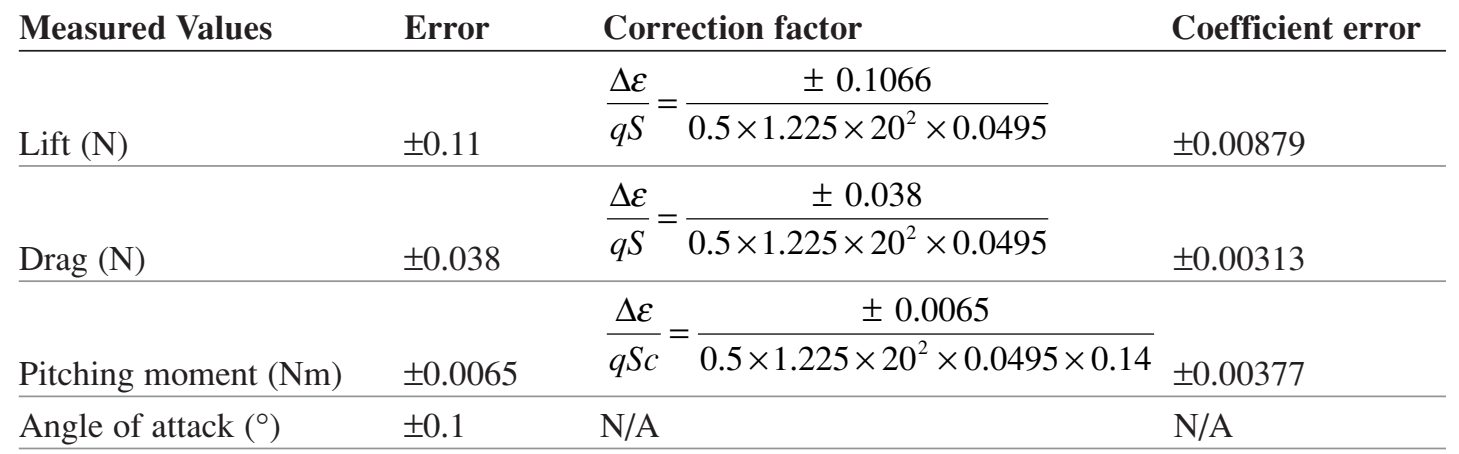

\subsection{Force Balance Measurements}

As initially described, the force balance measures fore and aft forces as well as the drag force through flexible cables attached to load cells and the output amplified by strain gauge amplifiers on the mounting support. The lift force is the sum of the fore and aft forces measured by the load cells for different angles of incidence minus the weight of the tested model, i.e. Lift $(L)=$ Weight $-[$ Fore + Aft $] \mathrm{N}$. The fore and aft forces are positioned either side of the centre of gravity of the model $(63.5 \mathrm{~mm})$ and therefore the lift coefficient, $C_{L}$, is calculated by

$$
C_{L}=\frac{L}{0.5 \rho V^{2} S_{w}}
$$

Where $0.5 \rho V^{2}=q$ is the dynamic pressure based on calibrated airspeed corrected for wind tunnel errors and $S_{w}$ is the wing area.

The pitching moment is the difference between the fore and aft forces as registered by the load cells multiplied by $0.127 \mathrm{~m}$, which is the distance between the load cells, i.e. Pitching Moment $(\mathrm{M})=$ $[$ Fore - Aft $] \times 0.127 \mathrm{Nm}$. 
Where the pitching moment coefficient, $C_{M}$, is given by

$$
C_{M}=\frac{M}{0.5 \rho V_{C A S}^{2}{ }_{C S} S_{w}}
$$

The drag force acting on the model is taken directly from the output of the load cells lying in the horizontal plane in the mid-section of the stem of the support. The drag coefficient which is the sum of the parasite drag and the lift induced drag is calculated by dividing the drag force by the dynamic pressure and the wing area for a tunnel speed of $20 \mathrm{~ms}^{-1}$ which is later on corrected for tunnel errors for the calibrated airspeed.

$$
\begin{gathered}
C_{D}=C_{D_{o}}+C_{D_{1}} \\
C_{D}=\frac{D}{0.5 \rho V_{C A S}^{2} S_{w}}
\end{gathered}
$$

\subsection{Surface Oil Flow Visualisation}

\subsubsection{Experimental Setup}

The tunnel test section is made up of Perspex walls giving extensive optical access to the test model which is mounted on the force balance support stem. The bottom wall of the test section is made from a porous board and therefore effort was made to cover the section with solid black card. As titanium powder was used, it was then possible to obtain bright images with a high contrast under normal light. A Camera kit section is mounted perpendicular to the test section on the top Perspex window. A Canon EOS Rebel DS126291 12.2 MP camera was used with a 18-55 mm lens.

\subsubsection{Test Requirements and Post-processing}

The wing of the model microlight was painted matt black so as to offer better contrast and brightness in image processing in studying the surface features. The oil-pigment mixture ratio was determined by a series of trial and error runs but generally involved suspending the titanium powder in paraffin and adding linseed oil to prevent clumping of the powder, as suggested by Lua [17]. Some of the titanium powder needed further fining and therefore grounding was done in the laboratory so as to have a mixture with uniform particle size. A mixture ratio of 1:3 was found to be adequate for titanium and paraffin respectively. Therefore for every $15 \mathrm{ml}$ of titanium dioxide, $45 \mathrm{ml}$ of paraffin was used with 1 $\mathrm{ml}$ of linseed oil. This mixture was such that, paraffin evaporates after approximately 10 seconds after the tunnel starts up, leaving behind streaks formed by the powdered titanium.

It is to be noted that, the temperature drop during the tunnel run will affect the viscosity of the oil used and therefore to some extent affect the results obtained. Therefore it is recommended to use both videos and stills so as to identify surface singularities that might not be captured in the final stills. The camera kit is mounted perpendicular to the surface and placed where there is extensive optical access to the model. Image processing is done using Microsoft Photo Gallery and JPEG photo recovery to help in identifying and tracing streak lines.

\subsubsection{Surface Oil Flow Visualisation Errors}

Due to pressure imbalance between the top and bottom surfaces of the wing, span wise flow of air can be observed leading to a difference between the local flow and the free stream flow. This is more common in swept wings than in un-swept wings. This flow might lead to lack of uniformity of the streaks formed on the wing surface. Other errors include changes in the ambient temperature leading to a change in the viscosity of the fluid used and acceleration effect resulting from accelerating the tunnel from an initial state with a speed of $0 \mathrm{~m} / \mathrm{s}$ to the required speed. This acceleration effects might lead to the formation of undesirable features on the wing surface including early separation and recirculation features resulting from low velocities. Therefore video imaging is recommended so as to identify surface singularities that might result from the above mentioned errors. 


\section{RESULTS}

\subsection{Oil Flow Visualisation}

On the clean wing oil flow pattern at 0 degree angle of attack (Figure 15a), it is observed that the streaks were fairly parallel with each other, with the local flow slightly offset from the free stream flow direction resulting from the pressure gradients (3D aerofoil) effects [18]. Flow over the top surface (upper theory) is observed to be inwards (cross flow) towards the wing root as indicated by the streaks due to the pressure over the top surface being less than the bottom surface (lower theory) and therefore causing a slight inward span wise flow towards the root (3D aerofoil effect). Separation is seen to occur at the trailing edge and marked by the dark region.

With the turbulators applied (Figure 15b), the streak lines are seen to be fairly aligned with the flow direction. Along the vertex of the turbulators, a stream of vortices is produced leaving the vertex at an angle equivalent to the sweep angle of the zigzag turbulator strips. Separation is observed to be at the trailing edge marked by the darkened region with wave like features with their maximum points aligned with the vertex or the point of origin of the vortices and the minimum or sagged points aligned with the vortices.
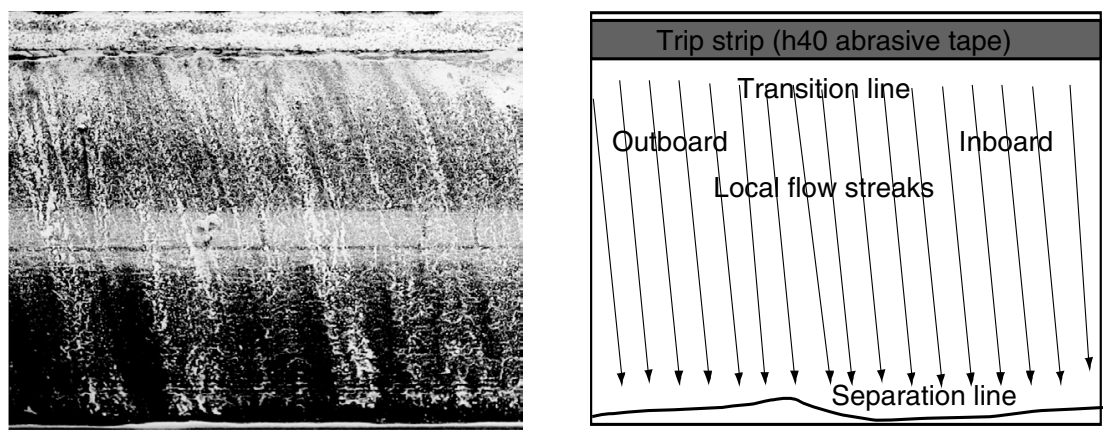

(a) Clean wing
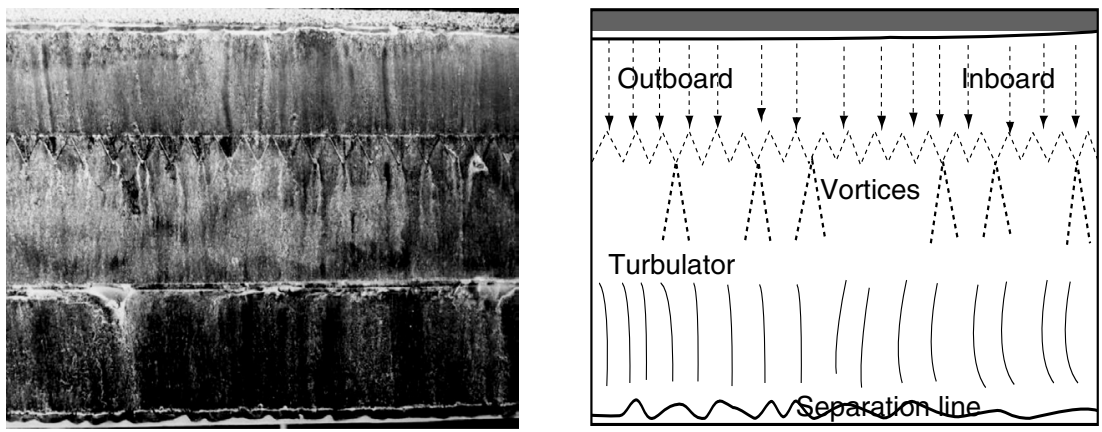

(b) Wing with turbulators
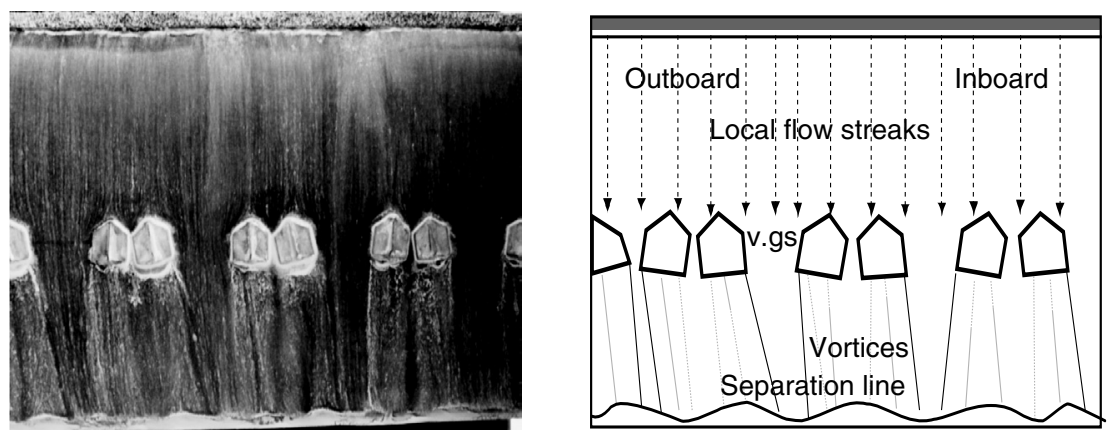

(c) Wing with vortex generators

Figure 15. Oil flow visualisation at $\alpha=0^{\circ}$. 
With vortex generators applied (Figure 15c), transition is seen to occur at the leading edge along the trip-strip position. This is followed by a sweep of the mixture, marking a turbulent flow, depriving the region with titanium powder and leaving dark streaks streaming along the flow direction. Past the vortex generators a series of stream wise vortices is seen to be produced shifting the separation region further downstream towards the trailing edge. This action leads to the accumulation of titanium powder at the trailing edge of the wing.

With a clean wing at $10^{\circ}$ (Figure 16a) further downstream, the streaks are seen to be parallel to each other. Along the separation region, less powder was observed marking high sweep.

As the action of the turbulator had been described earlier, it was observed that at $10^{\circ}$ (Figure 16b), the inboard section was beginning to experience backward flow marked by re-circulation zones, while the outboard separation was still at the trailing edge marked by diverging streaks on the outboard section of the wing. This condition was due to the $1^{\circ}$ of twist applied to the wing section in the earlier stages of design leading to a lower angle of attack for the outboard section than the inboard section.

With the vortex generators applied (Figure 16c) it was observed that, the vortices produced pushed the separation line further downstream and areas which lacked direct influence of vortices dipped
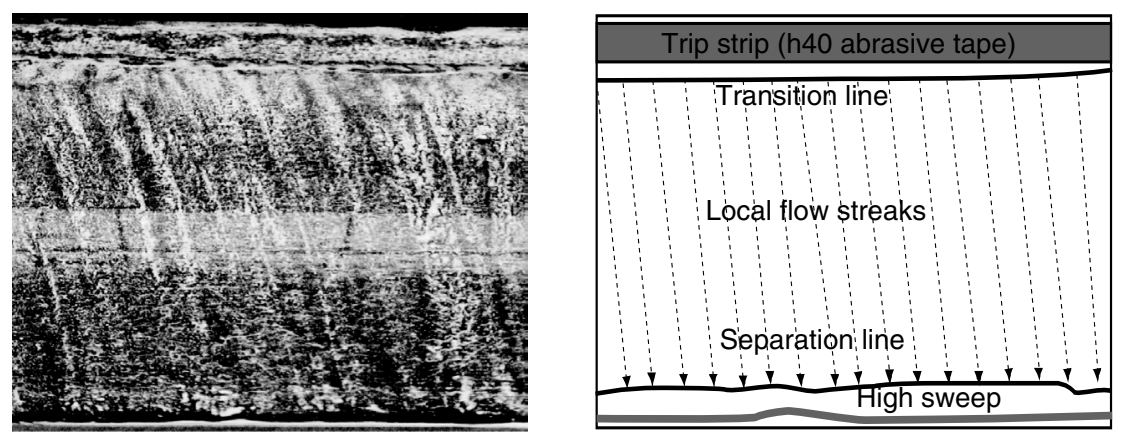

(a) Clean wing
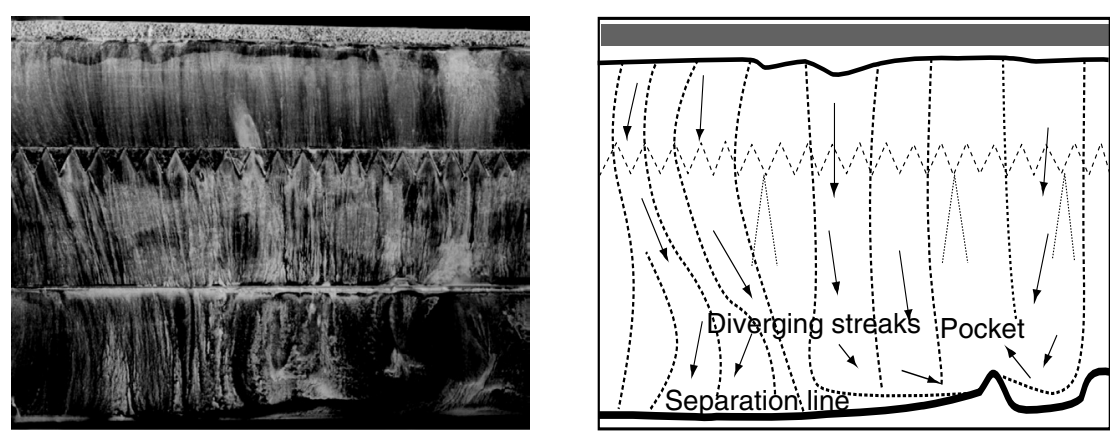

(b) Wing with turbulators
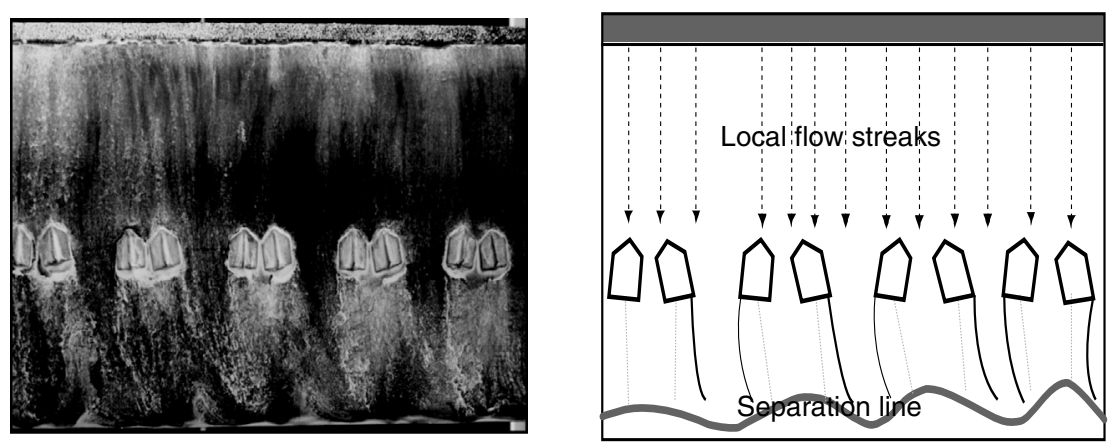

(c) Wing with vortex generators

Figure 16. Oil flow visualisation at $\alpha=10^{\circ}$. 
further upstream. This led to wave like features at the trailing edge of the wing. Also vortex breakdown started occurring in the inboard section marked by accumulation of titanium powder just downstream of the vortex generators attachment locations for the inboard section. As earlier seen with the turbulators that, at this angle of attack, the inboard section experienced recirculation zones and therefore the inboard vortex generators were influenced by this effect.

As from the theoretical and force balance analysis, it was established that at about $17^{\circ}$, the clean wing had completely stalled. Therefore at about $15^{\circ}$ (Figure 17a) the clean wing had stalled forming dips and recirculation zones along the span of the wing. Some chord wise flow was seen for the outboard section closer to the leading edge but no significant flow was observed at the trailing edge of the wing. The streaks at the leading edge are seen to converge initially then diverge towards the trailing edge.

With the turbulators applied (Figure 17b), it was observed that at $15^{\circ}$, the inboard section was completely stalled with streaks moving inwards from the outboard section forming the recirculation dips. Also a boundary for the backward flow was observed for the inboard section close to the attachment region of the turbulator.
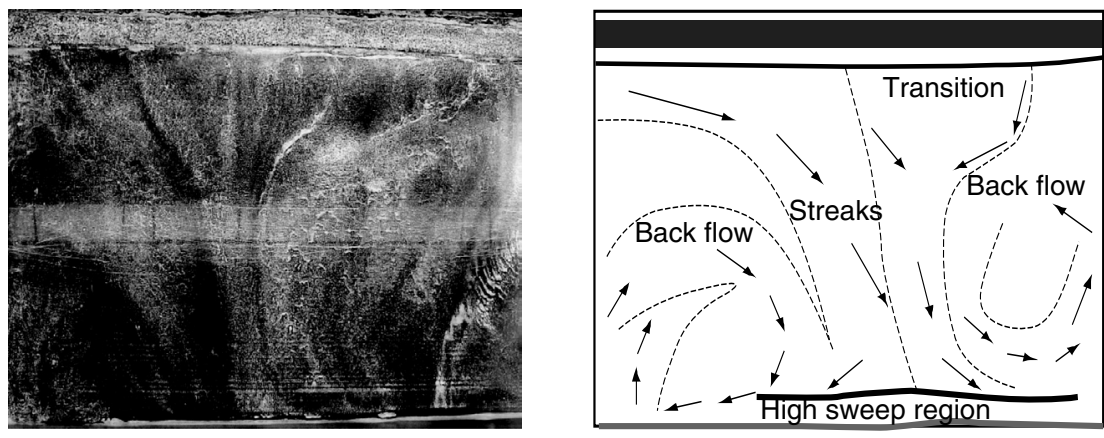

(a) Clean wing
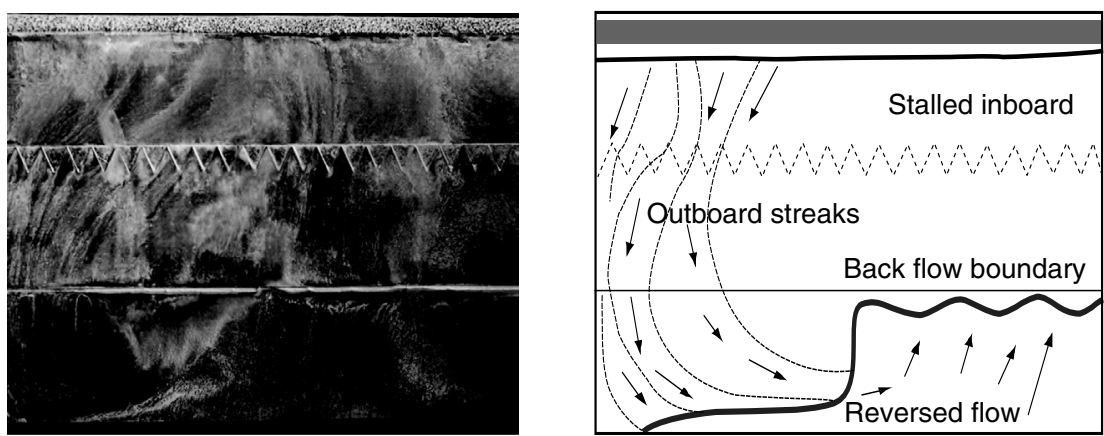

(b) Wing with turbulators
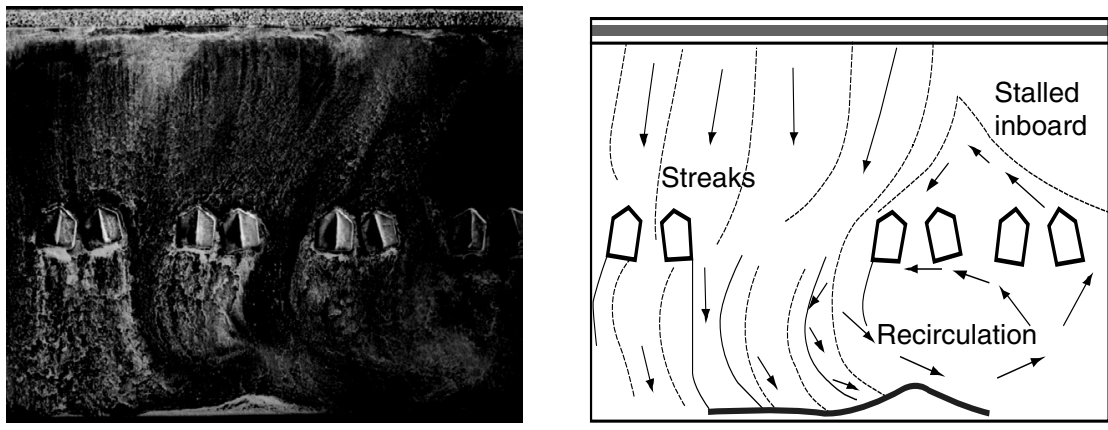

(c) Wing with vortex generators

Figure 17. Oil flow visualisation at $\alpha=15^{\circ}$. 
With the vortex generators applied (Figure 17c) it was observed that, the inboard section of the wing had stalled completely at $15^{\circ}$. Inward flow movement from the outboard section was observed by the curved vortex structures. Also the vortex generators in the inboard section were seen not to be effective at this angle of attack, as there weren't any vortices produced showing that they were inside the stall region (vortex breakdown).

\subsection{Force Balance Data}

The following results display the observed variation in lift coefficient with angle of attack for the microlight with and without the flow control devices from wind tunnel testing. The results show different properties in terms of the lift coefficient i.e. $C_{L_{\max }}, C_{L_{\alpha}}, C_{L_{0}}, \alpha_{\text {critical }}$ and other properties calculated as derivatives of the primary ones.

\subsubsection{Lift Curve}

For the clean model, the observed $C_{L_{\max }}$ was 1.09 at $\alpha_{\text {critical }}=17^{\circ}$. With MTOW $=450 \mathrm{~kg}$, a stall speed of $19.6 \mathrm{~m} / \mathrm{s}$ or $36.3 \mathrm{knots}$ is calculated. The observed $C_{L_{0}}$ was 0.29 , which corresponds to a lift curve slope, $C_{L_{\alpha}}=0.0896 /{ }^{\circ}$. Also the model was observed not to produce any lift at approximately $-3^{\circ}$ as seen in Figure 18.

With the turbulators applied on the model-microlight, the observed $C_{L_{\max }}$ was $1.12 ; 2.8 \%$ higher than on the clean wing at $\alpha_{\text {critical }}=20^{\circ}$, marking a $17.6 \%$ increase from the initial stall angle of $17^{\circ}$ on the clean wing. The stall speed corresponding to $C_{L_{\max }}=1.12$ is observed to be $19.33 \mathrm{~ms}^{-1}$ or 35.8 knots which is $1.4 \%$ lower than the observed stall speed with a clean wing. It was also observed that the turbulators had no significant influence on $C_{L_{\alpha}}$. Having similar lift curve slopes, the observed lift coefficient at zero incidence, $C_{L_{0}}$, and zero lift angle of attack were 0.29 and $-4^{\circ}$ respectively, similar to the values of the clean wing. It was also observed that the turbulators made the stall more gradual than with a clean wing. With every $1^{\circ}$ after the critical angle of attack, the estimated average drop in the lift coefficient was $4 \%$ with a clean wing while with the turbulators applied, it was $1.8 \%$ for angles of attack in the range of $20-25^{\circ}$. It was noted that the turbulators had no significant influence on the lift at lower angles of attack but had significant influence on the lifting ability of the wing at higher angles of attack.

With vortex generators applied on the wing surface, the observed $C_{L_{\max }}$ was $1.05,3.7 \%$ lower than with a clean wing at $\alpha_{\text {critical }}=18^{\circ}$. This corresponded to a stall speed of $20 \mathrm{~ms}^{-1}$ or $37 \mathrm{knots} ; 2 \%$ higher than the stall speed of the clean wing. The observed $C_{L_{0}}$ and was 0.21 , giving a zero lift angle of attack of $-3^{\circ}$. The vortex generators had a significant influence on the lifting ability of the wing at all angles

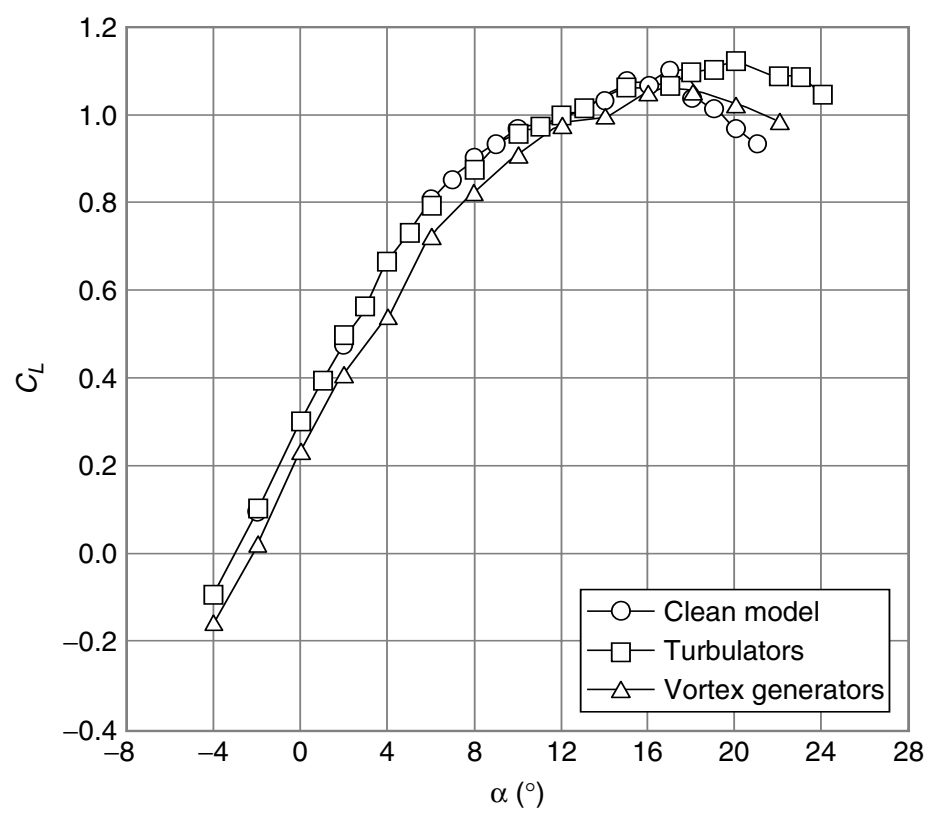

Figure 18. Lift curve slope for the model with and without the flow control devices.

Volume $5 \cdot$ Number $3+4 \cdot 2013$ 
of attack. With the vortex generators applied, a gradual loss in lift was observed post-stall. For every $2^{\circ}$ increase in the angle of attack after, the average drop in the lift was observed to be $3 \%$ which was more gradual than with a clean wing of which the average drop was $4 \%$ for every $2^{\circ}$. The stall speed with vortex generators was observed to be $3.5 \%$ higher compared to the stall speed with turbulators applied. Overall, the lift generated with vortex generators applied was lower than the lift generated with the turbulators for lower and higher angles of attack.

Generally the lift observed with and without the devices was lower compared to the 3D and 2D analysis carried out in XFLR5, which suggested a $C_{L_{\max }}$ of 1.3 giving a $15 \%$ difference between the values. This $2 \mathrm{D}$ value is equivalent to $3 \mathrm{D} C_{L_{\max }}=1.2$ and is seen to be $8.3 \%$ higher than the $C_{L_{\max }}$ of a clean wing, which is an acceptable range for correlation.

\subsubsection{Drag Polar and Drag Curve}

From the drag polar the property of particular interest is the drag at zero lift, $C_{D_{0}}$ and/or the minimum drag, $C_{D_{\min }}$. Therefore a relation given below is used to relate the different forms of drag on the aircraft

$$
C_{D}=C_{D_{\min }}+k\left(C_{L}-C_{L_{o}}\right)^{2}
$$

For this wing, $C_{D_{o}}$ is not exactly equal to $C_{D_{\min }}$ due to the presence of camber thus assuring lift production even at the minimum drag level. This tends to increase the lift-to-drag ratio. $C_{D_{\min }}$ is termed the parasite drag and is dependent on the flight Mach number, Reynolds number and configuration. The effect of $C_{D_{o}}$ is to shift the drag polar upwards.

For the clean wing configuration (Figure 19), $C_{D_{o}}=0.051$. With the turbulators applied, $C_{D_{o}}=0.047$; $8 \%$ lower than the clean wing. With vortex generators applied, $C_{D_{o}}=0.055 ; 8 \%$ higher than the clean wing. For the section where the drag is dependent on the lift, the turbulators are seen to produce, on average, a high lift value compared to vortex generators for a given amount of drag. The induced drag comes from the induced angle of attack as a secondary effect of lift generation and therefore is directly related to the lift. This comes from the wing tip vortices arising from the span-wise flow around the tip and over the top-surface due to pressure imbalance. These tip-vortices tend to reduce the effective angle of attack due to the downwash effect. The induced angle of attack from the downwash effect leads to the production of the induced drag from the lift force produced. Since the induced drag, $k C_{L}^{2}$, is directly proportional to the lift force, the turbulators at higher angles of attack are seen to generate more induced drag on the model than with a clean wing or with vortex generators applied due to high lift production. The drag polar can be related to the drag curve shown in Figure 20, showing the variation of drag with the angle of attack.

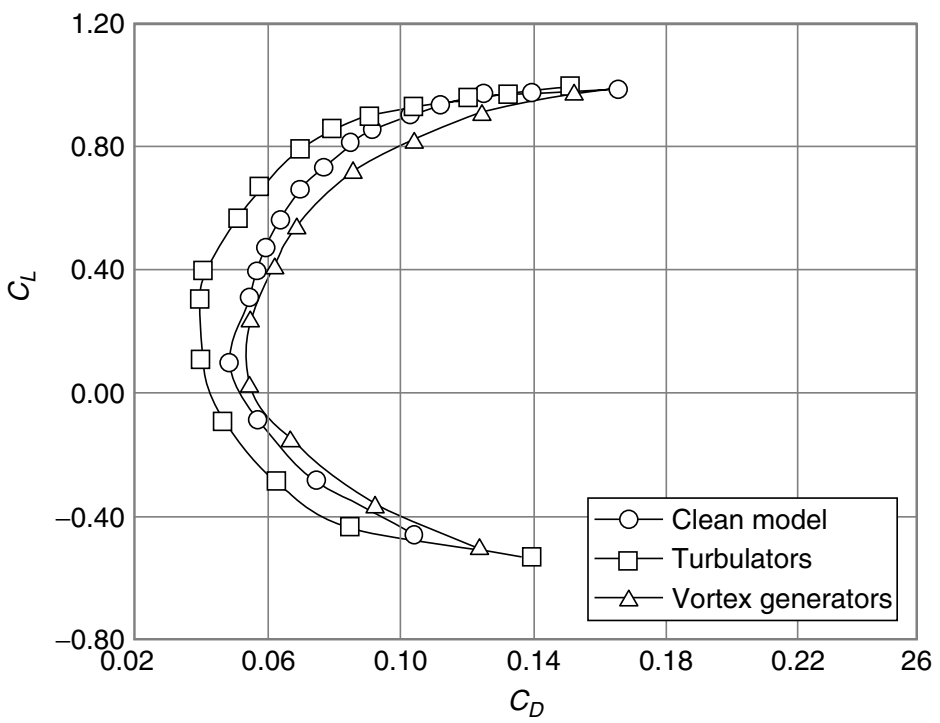

Figure 19. Drag Polar showing the lift coefficient and the drag coefficient with and without flow control devices. 
It can generally be observed that, the drag of the microlight tends to increase with increasing angle of attack. At about $12^{\circ}$ the variation is seen to increase with more drag generation for every increment in the angle of attack. The increment in the drag penalty at higher angles of attack is due to the increased lift dependent and pressure drag due to increased adverse pressure gradient. Both vortex generators and turbulators are observed, at angles of attack $12-20^{\circ}$, to decrease the drag penalty (Figure 20). These devices tend to produce vortices which tend to move the separation region further downstream by mixing the high momentum fluid to the low momentum fluid in the lower regions of the boundary layer. These vortices also tend to reduce the separation velocity and increase the back pressure and therefore reduce the adverse pressure gradient.

It is also observed that, the drag reduction at higher angles of attack closer to stalling is greatest with vortex generators than with turbulators applied. This might have been due to a higher lift dependent drag component with the turbulators applied at these angles, resulting from high lift generation. Therefore the vortex generators become effective by generating less skin friction and lift-induced drag while inducing a considerable reduction in the pressure drag. Since the turbulators were designed below the critical roughness height, their efficiency in terms of drag reduction was observed at lower angles of attack. With turbulators applied the skin friction drag generation at lower angles of attack was less (less device drag) compared to the increase in surface static pressure and therefore reducing the adverse pressure gradient. The turbulators, being manufactured out of nylon-film had a smoother surface finish compared to the model wing which was made out of polyurethane and had a spray painted coating. In addition, the turbulators have a width-to-chord ratio of $0.3(30 \%)$ and a thickness-to-chord ratio of only $1 \times 10^{-3}(0.1 \%)$. Therefore, the turbulators are expected to play a significant role in reducing the skin friction coefficient at lower angles of attack relative to the clean wing.

\subsubsection{Lift-to-drag Ratio}

Furthermore the benefits of drag reduction for different configurations i.e. higher and lower angles of attack with vortex generators and turbulators are further discussed using the lift-to-drag ratio (Figure 21). With a clean wing configuration, the maximum lift-to-drag ratio, $L / D$, was 9.5 at $\alpha=5^{\circ}$. With the turbulators applied, $L / D=11.6$ at $\alpha=4^{\circ}$ and is $22 \%$ higher than the clean wing. With the turbulators applied, the skin friction drag generation at lower angles of attack is less compared to the lift production. With the vortex generators applied, the observed $L / D=8.4 ; 11.6 \%$ lower than the clean wing. This is due to more drag generation (device drag) at lower angles of attack. The drag at lower angles of attack is mainly skin friction drag due to the vortex generators acting as surface protuberances. For higher angles of attack, i.e. $12-20^{\circ}$, the effect of the vortex generators and the

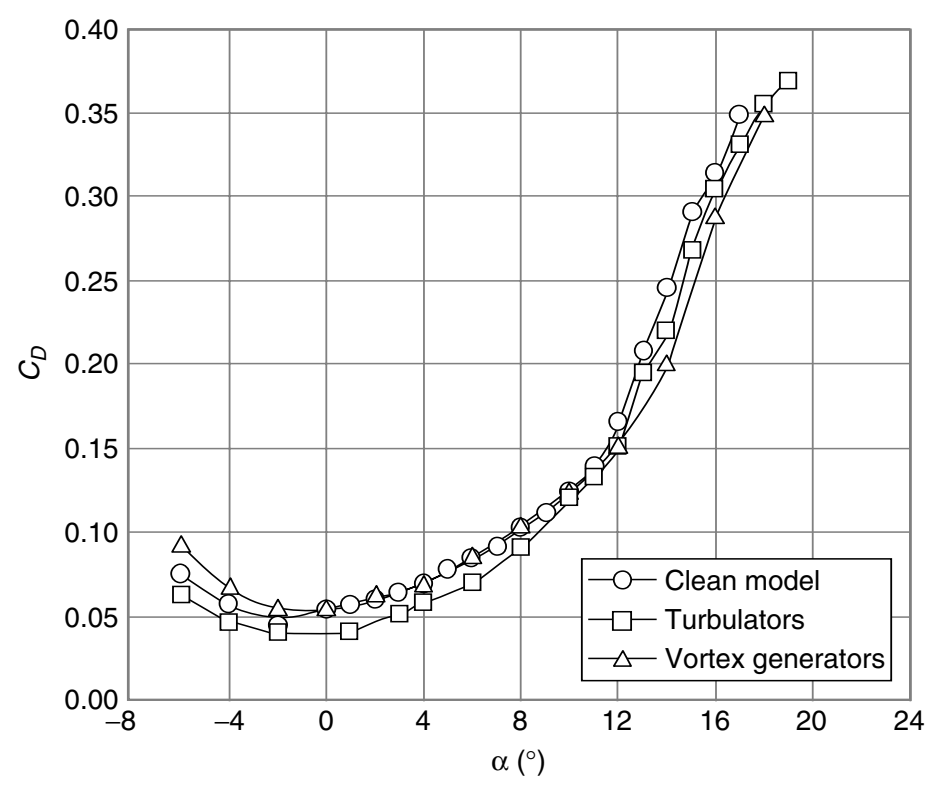

Figure 20. Drag curve showing the variation of drag with angle of attack. 


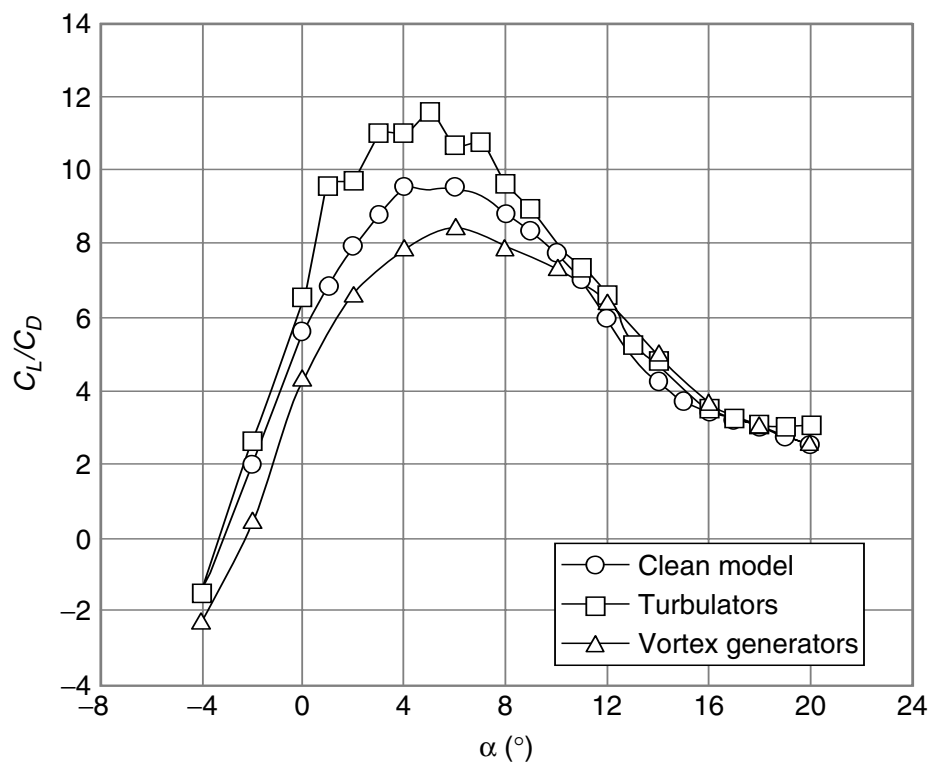

Figure 21. Lift-to-drag ratio variation with angle of attack with and without the flow control devices.

turbulators on $L / D$ is comparable. As earlier described, the drag at higher angles of attack is mainly dominated by the pressure drag due to the increasing adverse pressure gradient and the lift dependent drag and therefore the effect of the skin friction drag becomes less pronounced for both the turbulators and vortex generators.

\subsubsection{Pitching Moment Coefficient}

The pitching moment forms the basis of understanding the longitudinal stability of the aircraft. A negative gradient of the pitching moment is desired with the angle of attack for longitudinal stability. This variation is such that the pitching moment tends to decrease with increasing angle of attack. This variation of the pitching moment ensures that a restoring moment is produced tending to restore the aircraft to its equilibrium position after a change in the angle of attack. To ensure a positive longitudinal stability then the centre of gravity is located forward of the stick fixed neutral point of the aircraft. For this particular configuration, the tail section plays a vital role to ensure that the net moment produced tends to cause a restoring moment bringing the aircraft back to its equilibrium point after an initial disturbance. Therefore the results in Figure 22 indicate the variation of the pitching moment coefficient with the angle of attack for the model-microlight with and without the flow control devices. The reference location is taken to be the pivot/force balance connection point which is the estimated centre of gravity for the model.

Generally the pitching moment variation with the angle of attack, $C_{M_{\alpha}}$, is seen to ensure a state of positive longitudinal stability with and without the flow control devices. The estimated $C_{M_{\alpha}}$ with a clean wing is $-0.0225 /^{\circ}$; with the turbulators applied $C_{M}=-0.0214 /{ }^{\circ}$ and with the vortex generators applied $C_{M_{\alpha}}=-0.0176 /{ }^{\circ}$. It is generally noted that the turbulator strip has no significant influence on the longitudinal stability of the aircraft at higher angles of attack and therefore retains the same longitudinal stability pattern as with the clean model. The vortex generators, however, have decreased the magnitude of the pitching moment variation with angle of attack by $22 \%$. This effect is mainly observed at higher angles of attack by a reduction in the magnitude of the pitching moment coefficient. It is also noted that the more forward the centre of gravity from the aerodynamic centre the more stable the aircraft is but it is not to be too far forward. Since the estimated centre of gravity is very close to the leading edge, adding weights like vortex generators aft of this location would tend to move the centre of gravity slight aft of the estimated position and resulting into a decreased longitudinal stability. This implies that, closer to stall conditions, with vortex generators applied, the longitudinal stability is reduced compared to a clean wing configuration or compared to using turbulators 


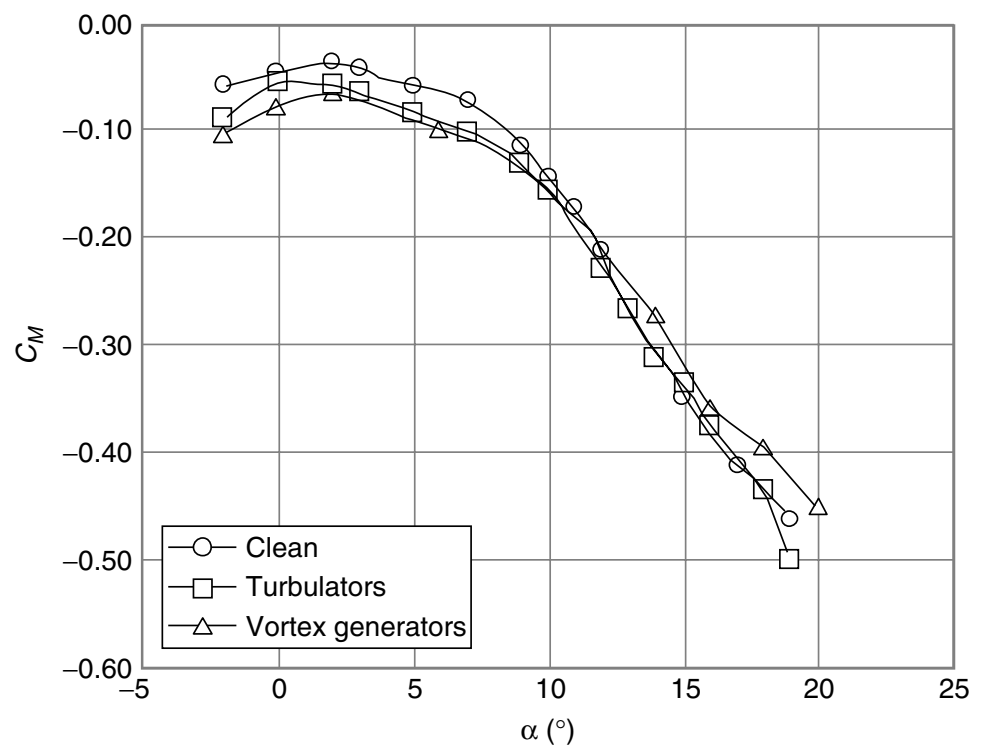

Figure 22. Pitching moment variation with the angle of attack with and without the flow control devices.

\section{DEVICE APPLICATION ON THE FULL-SCALE AIRCRAFT}

\subsection{Device Efficiency}

Since the devices tend to enhance the stall characteristics by the use of vortices, the performance parameter of these devices is taken as the ratio between the strength of the vortices produced as a function of the height of the device and the free stream velocity and the device drag. This ratio is seen to be directly proportional to the lift-to drag-ratio of the device as suggested by ESDU [6]. Therefore a plot of $L / D$ for the devices compared with the clean wing configuration is presented in Figure 23 .

Generally, at lower angles of attack, with the turbulators applied, the performance parameter is generally higher compared to the vortex generators suggesting less device drag and increased device efficiency than the vortex generators. The average performance parameter is $+25 \%$ greater with the turbulators applied for angles of attack ranging from $0-10^{\circ}$. With the vortex generators, the drag

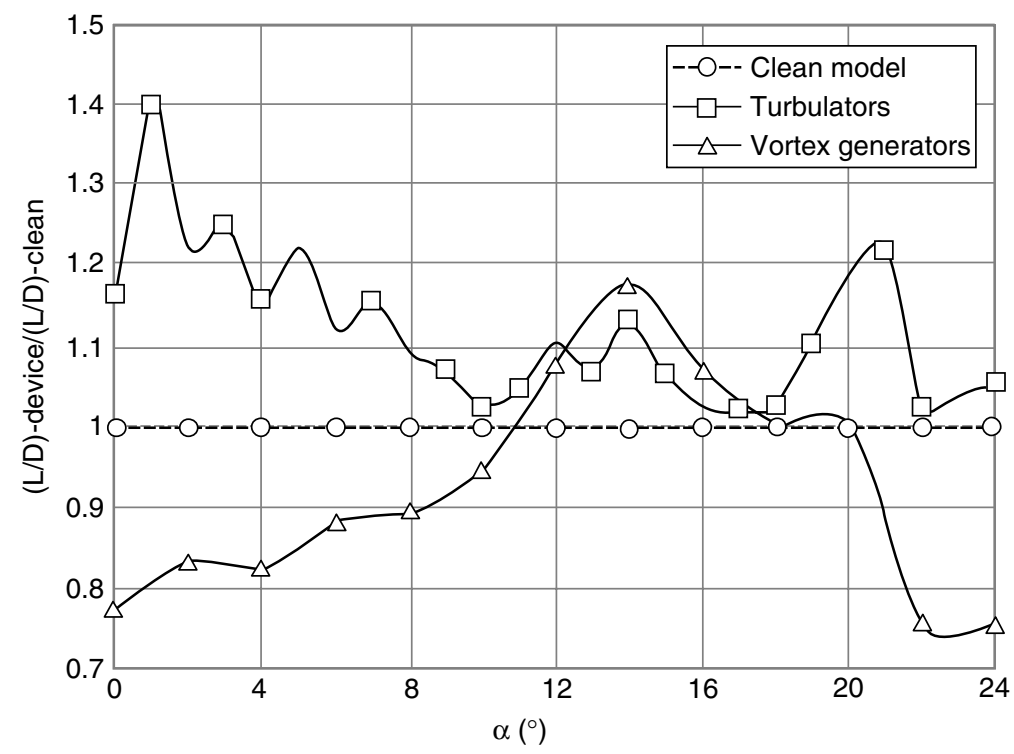

Figure 23. A device performance parameter in terms of the lift to drag ratio. 


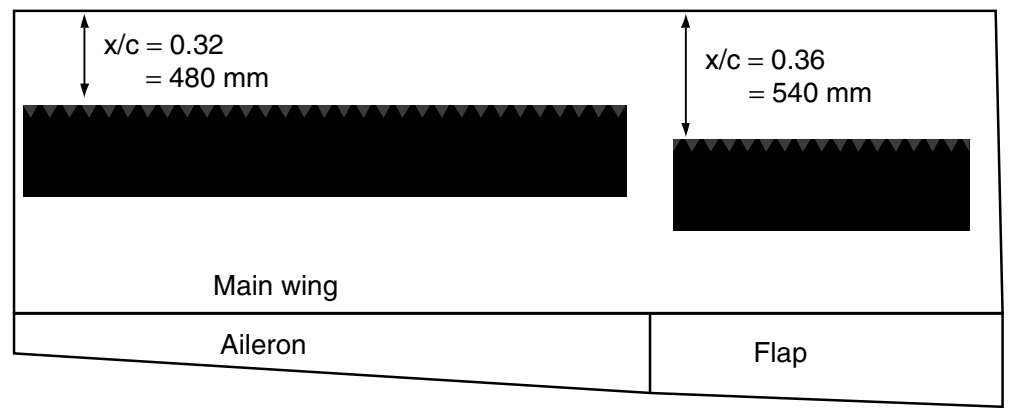

Figure 24. Chord wise turbulator position on the microlight wing (not to scale).

penalty is seen to be greater than the lift augmentation with an average performance parameter of $-14 \%$. This is mainly due to the small boundary layer thicknesses at lower angles of attack. This leads to the vortex generators acting as surface protuberances and therefore reducing their effectiveness. With an increase in the angle of attack, the turbulators are seen to show a gradual decrease in their performance, whilst an increase in performance is observed of the vortex generators (up to about $15^{\circ}$ ) with a $+17 \%$ positive performance parameter. At this angle of attack, the observed performance parameter with the turbulators is observed to be slightly lower at $+14 \%$. Overall, the efficiency of the turbulators is seen to be higher at lower and higher angles of attack.

\subsection{Optimum Position}

Therefore for an actual application of passive flow control on the microlight, the turbulators are recommended. Applying the turbulators will lead to an increase in the safety margin of the microlight. Surface oil flow visualisation revealed different boundary layer behaviour between the inboard and outboard sections due to the geometric twist induced earlier in the manufacturing of the model. On a practical basis, trailing edge vortices usually introduce a downwash which tends to change the angle of attack, such that the inboard and outboard sections are at different angles of incidence as earlier described.

For a free stream Reynolds number of $\sim 2$ million, based on a mean aerodynamic chord $=1.5 \mathrm{~m}$ and a flight speed of $30 \mathrm{~ms}^{-1}$, the turbulators are required to be placed at a chord wise position equivalent to $\mathrm{x} / \mathrm{c}=0.36$ or $0.54 \mathrm{~m}$ from the leading edge of the microlight wing. This position is $10 \%$ ahead of the estimated separation region at $17^{\circ}$ angle of attack and does not take into account the $1^{\circ}$ of twist (washout). For the case of having a twist, a washout of $1^{\circ}$ towards the tip, then the tip stalls at a higher angle of attack than the root, i.e. $18^{\circ}$. At this angle, the separation region lies at a chord wise location of $\mathrm{x} / \mathrm{c}=0.37$. Therefore the turbulators should be located $10 \%$ ahead of this point, i.e. $\mathrm{x} / \mathrm{c}=0.32$ for optimum performance. The estimated positions of the turbulators for the inboard and outboard section of the wing are shown in Figure 24.

\section{CONCLUSIONS}

This paper sets out an experimental investigation of passive flow control devices - vortex generators and turbulators, as a means to enhance the stall characteristics of a microlight aircraft. The work comprises computational analysis (Javafoil and XFLR5) to support design and scaling of the flow control devices and subsequent experimentation in a wind tunnel with surface oil flow visualisation and force balance measurements. Analysis of the results obtained from the force balance measurements produced key outputs including lift coefficient and pitching moment coefficient curves, drag polar and lift-to-drag ratio. Experiments were run with and without the flow control devices to provide a clean wing baseline for comparison

- Results indicated improved performance at lower drag penalty with the turbulators applied than with the vortex generators.

- With the turbulators applied, the stall speed of the aircraft was lowered by $1.4 \%$ and the critical angle of attack increased from $17^{\circ}$ on a clean model to $20^{\circ}$. With vortex generators applied, a delay in stall was observed by a $2 \%$ increase in the critical angle of attack and reduced the drag penalty at higher angles of attack. 
- $\quad$ Positive longitudinal stability was observed on the model with and without the flow control devices. With the vortex generators installed, the longitudinal stability was slightly reduced particularly at higher angles of attack compared to turbulators, which maintained approximately a similar stability pattern to that of a clean model with some increased stability at lower angles of attack.

- A greater comparison is offered between the model with and without the devices so as to determine the most suitable flow control device for application. The turbulators offer a greater degree of aerodynamic efficiency (lift-to-drag ratio) than the vortex generators across a range of operational angles of attack and therefore are recommended for application on the microlight aircraft.

\section{NOMENCLATURE}

$A R_{v}=$ Vortex generator aspect ratio

$c \quad=$ Wing mean aerodynamic chord

$C_{L} \quad=$ Aerofoil (2D) or wing (3D) lift coefficient

$C L_{v}=$ Delta wing lift coefficient

$C L_{a}=$ Lift curve slope

$C_{D}=$ Aerofoil (2D) or wing (3D) drag coefficient

$C_{M}=$ Aerofoil (2D) or wing (3D) pitching moment coefficient

$C_{M_{0}}=$ Pitching moment coefficient at the aerodynamic centre

$d_{v}{ }_{D}=$ Distance between vortex generators of the same pair

$D_{v} \quad=$ Distance between a pair of vortex generators

$h=$ Vortex generator height

$h_{n} \quad=\quad$ Static margin

$H_{32}=$ Shape factor

$k=$ Drag due to lift factor

$K=$ Grit roughness constant; Vortex generator strength

$K_{v} \quad=\quad$ Vortex generator strength parameter

$K_{p}^{\prime} \quad=\quad$ Vortex generator lift constant for a profile flow

$K_{v}{ }^{\prime}=$ Vortex generator lift constant for viscous flow

$\operatorname{Re}=$ Reynolds number

$S=$ Wing area

$S_{v} \quad=\quad$ Vortex generator area

$V_{s} \quad=\quad$ Stall speed

$V \quad=$ Free stream velocity

$W \quad=$ Weight

$\alpha=$ Wing angle of attack

$\beta=$ Vortex generator incident angle

$\delta=$ Boundary layer thickness

$\delta_{1}=$ Boundary layer displacment thickness

$\delta_{2}=$ Boundary layer momentum thickness

$\delta_{3}=$ Boundary layer energy thickness

$\rho=$ Density

$\Lambda=$ Sweep angle

$\mu \quad=$ Dynamic viscosity

$v \quad=$ Kinematic viscosity

\section{LIST OF ACRONYMS}

AoA Angle of Attack

AC Aerodynamic Centre

A/C Aircraft

CG Centre of Gravity

CAD Computer Aided Design

MAC Mean Aerodynamic Chord

MTOW Maximum Take-Off Weight

VLM Vortex Lattice Method

2D Two-Dimensional

3D Three-Dimensional 


\section{REFERENCES}

[1] BMAA. British Microlight Aircraft Association. [Online] 0408 2013. http://www.bmaa.org/.

[2] Langley Research Centre. Micro-Vortex Generators Enhance Aircraft Perfomance. NASA. [Online] 0408 2013. http://www.nasa.gov/centers/langley/news/factsheets/Micro-VG.html.

[3] Air creation. Wings - Bionix 15. Air Creation in Australia. [Online] 04082013. http://www.aircreation.com.au/24-bionix-15.html.

[4] Pope, A., Rae, W.H. and Barlow, J.B. Low Speed Wind Tunnel Testing. USA : John Wiley \& Sons Inc., 1999.

[5] Hepperle, M. Turbulators. MH-AeroTools. [Online] 1602 2008. http://www.mhaerotools.de/airfoils//jf_validation.htm.

[6] XFLR5, Results vs Prediction. [Online] 10 2009. http://www.xflr5.com/docs/ Results_vs_Prediction.pdf

[7] Velte, C.M., Hansen, M.O.L. and Cavar, D., 2008. Flow analysis of vortex generators on wing sections by stereoscopic particle image velocimetry measurements. Environmental Research Letters, Vol. 17, p. 11.

[8] Vortex generators for control of shock induced separation. ESDU. 04, 1993, Engineering Science Data Unit.

[9] Koike, M., Nagayoshi, T. and Hamamoto, N., 2004. Research on Aerodynamic Drag Reduction by Vortex Generators. Mitsubishi Motors Technical Review, Vol. 16, pp. 11-16.

[10] Lin, J.C., 2002. Review of research on low-profile vortex generators to control boundary layer separation., Progress in Aerospace Sciences, Vo. 38, pp. 389-420.

[11] ESDU, 1995. Vortex generators for control of shock-induced separation. Part 2: guide to use of vane vortex generators. Engineering Science Data Unit, 01 Feb. pp. 1-34.

[12] Selvam, S., Thiyagarajan, P. \& Suresh, S., 2013. Experimental Studies on Wire Coiled Coil Matrix Turbulators with and Without Centre Core Rod. Arabian Journal for Science and Engineering, 38(9), pp. 2557-2568.

[13] Shui, L., Gao, J., Shi, X. \& Liu, J., 2013. Effect of duct aspect ratio on heat transfer and friction in steam-cooled ducts with 60 degrees angled rib turbulators. Experimental Thermal and Fluid Science, 49(0894-1777), pp. 123-134.

[14] Hepperle, M. Turbulators. MH-AeroTools. [Online] 0408 2013. http://www.mhaerotools.de/airfoils/turbulat.htm.

[15] AFE. Gliding Equipment. Airplane Flight Equipment. [Online] AFE, 2013. [Cited: 2013.]

[16] Chong, T.P. Turbulent Intensity. West London : s.n., 2013.

[17] Lua, F.K., 2010. Surface Oil Flow Visualisation., The European Physical Journal, pp. 52-63.

[18] Tang, G., Simpsons, L.R. and Tian, Q., 2004. Flow Visualization on the nlinear compressor cascade endwall using oil flows and laser doppler anemometry. Measurement Science and Technology, Vol. 15, pp. 1910-1916. 